\title{
Physical therapies for reducing and controlling lymphoedema of the limbs (Review)
}

\author{
Preston NJ, Seers K, Mortimer PS
}

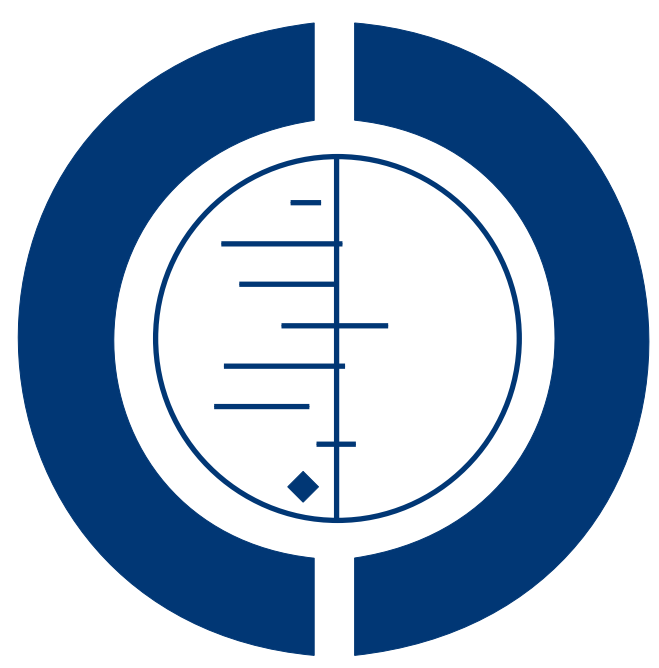

\section{THE COCHRANE COLLABORATION $^{\circledR}$}

This is a reprint of a Cochrane review, prepared and maintained by The Cochrane Collaboration and published in The Cochrane Library 2008, Issue 3

http://www.thecochranelibrary.com

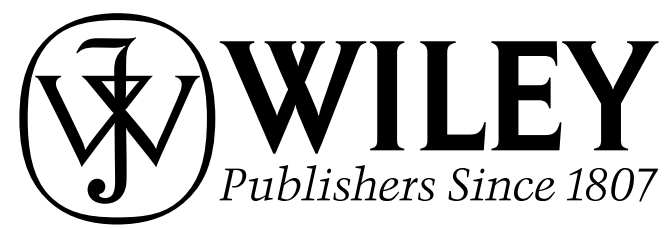

Physical therapies for reducing and controlling lymphoedema of the limbs (Review)

Copyright (C) 2008 The Cochrane Collaboration. Published by John Wiley \& Sons, Ltd. 
TABLE OF CONTENTS

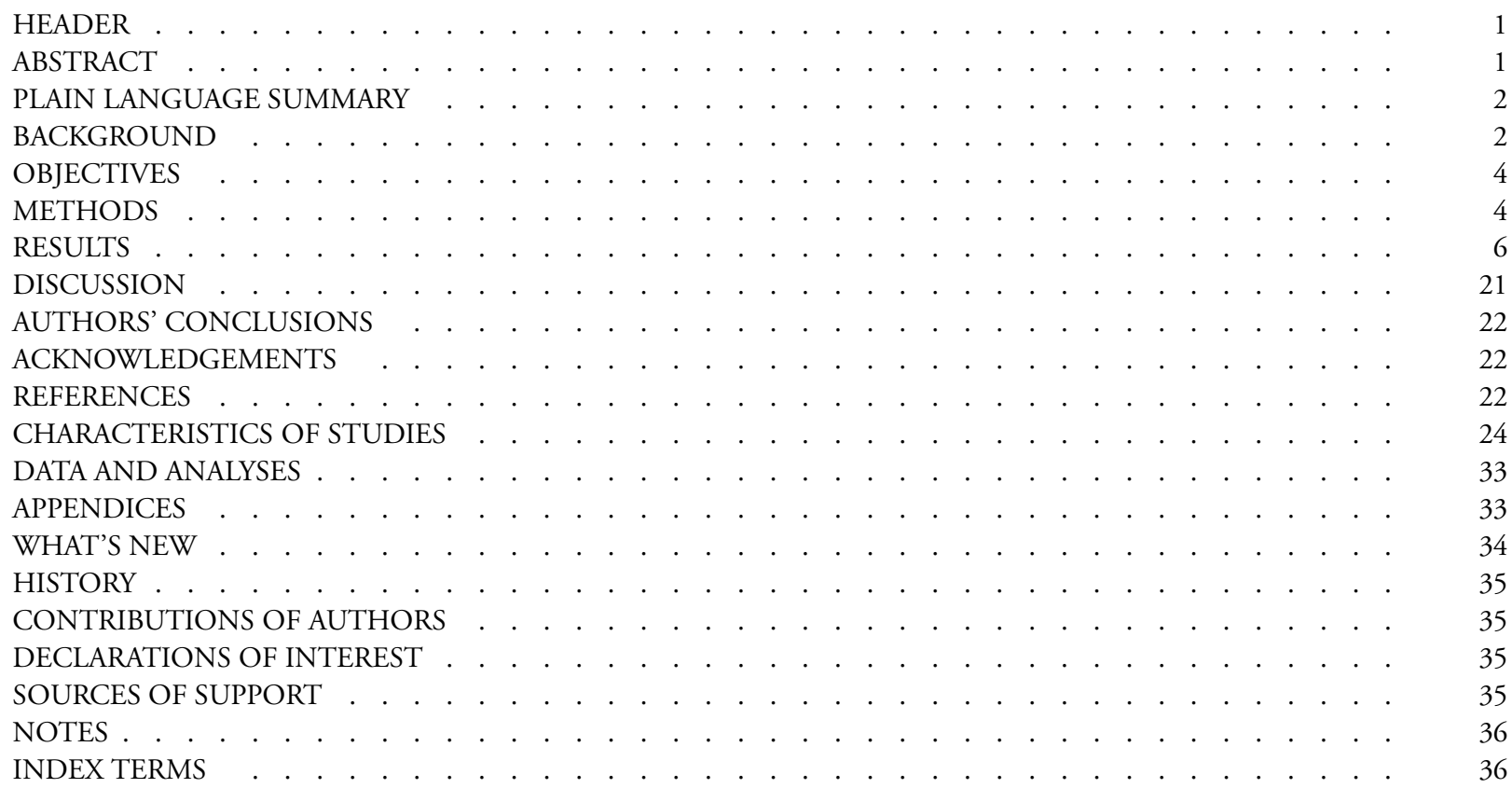




\section{Physical therapies for reducing and controlling lymphoedema of the limbs}

Nancy J Preston ${ }^{1}$, Kate Seers ${ }^{1}$, Peter S Mortimer ${ }^{2}$

${ }^{1}$ Research Team, RCN Institute, Oxford, UK. ${ }^{2}$ Division of Physiological Medicine, Cardiac and Vascular Sciences, St Georges Hospital Medical School, London, UK

Contact address: Kate Seers, Research Team, RCN Institute, Radcliffe Infirmary, Woodstock Rd, Oxford, OXON, OX2 6HE, UK. kate.seers@rcn.org.uk.

Editorial group: Cochrane Breast Cancer Group.

Publication status and date: New search for studies and content updated (no change to conclusions), published in Issue 3, 2008.

Review content assessed as up-to-date: 19 February 2008.

Citation: Preston NJ, Seers K, Mortimer PS. Physical therapies for reducing and controlling lymphoedema of the limbs. Cochrane Database of Systematic Reviews 2004, Issue 4. Art. No.: CD003141. DOI: 10.1002/14651858.CD003141.pub2.

Copyright (C) 2008 The Cochrane Collaboration. Published by John Wiley \& Sons, Ltd.

\section{A B S T R A C T}

\section{Background}

Lymphoedema is the accumulation of excess fluid in the body caused by obstruction of the lymphatic drainage mechanisms. Management involves decongesting the reduced lymphatic pathways in order to reduce the size of the limb. There is a great deal of debate as to which components of a physical treatment programme are the most crucial.

\section{Objectives}

To assess the effect of physical treatment programmes on:

volume, shape, condition and long-term control of oedema in lymphoedematous limbs;

psycho-social benefits.

\section{Search strategy}

We searched the Cochrane Breast Cancer Group trials register (October 2007), the Cochrane Central Register of Controlled Trials (The Cochrane Library Issue 1, 2008), MEDLINE, EMBASE, CINAHL and the National Research Register (February 2008) and UnCover, PASCAL, SIGLE, reference lists produced by The British Lymphology Society and The International Society of Lymphology congress proceedings (September 2003).

\section{Selection criteria}

Randomised controlled clinical trials that tested physical therapies with a follow-up period of at least six months.

Data collection and analysis

Two blinded reviewers independently assessed trial quality and extracted data . Meta-analysis was not performed due to the poor quality of the trials. 


\section{Main results}

Only three studies involving 150 randomised patients were included. Since none studied the same intervention it was not possible to combine the data. One crossover study of manual lymph drainage (MLD) followed by self-administered massage versus no treatment, concluded that improvements seen in both groups were attributable to the use of compression sleeves and that MLD provided no extra benefit at any point during the trial. Another trial looked at hosiery versus no treatment and had a very high dropout rate, with only 3 out of 14 participants in the intervention group finishing the trial and only 1 out of 11 in the control group. The authors concluded that wearing a compression sleeve is beneficial. The bandage plus hosiery versus hosiery alone trial, concluded that in this mixed group of participants bandage plus hosiery resulted in a greater reduction in excess limb volume than hosiery alone and this difference in reduction was maintained long-term.

\section{Authors' conclusions}

All three trials have their limitations and have yet to be replicated, so their results must be viewed with caution. There is a clear need for well-designed, randomised trials of the whole range of physical therapies if the best approach to managing lymphoedema is to be determined.

\section{PLAIN LANGUAGE SUMMARY}

\section{Physical therapies for reducing and controlling lymphoedema of the limbs}

Lymphoedema is the build up of excess fluid in the body tissues because of obstruction of lymphatic drainage back into the bloodstream. The affected limb becomes swollen, distorted in shape with pain, discomfort all of which impair movement and daily activities. It can be caused by a congenital abnormality, chronic venous insufficiency, damage to the lymphatic system following treatment of cancer or filariasis, a parasitic infection endemic in parts of India and Africa. Skin care is important as the affected tissues gradually thicken and are susceptible to inflammation and infections. People are also encouraged to exercise regularly and control their weight. Different physical treatments aimed at improved lymph drainage include multi-layer bandaging, manual lymph drainage (MLD), selfadministered massage and compression sleeves or hosiery.

The authors of this review, which aimed to assess the effect of physical treatment programmes on the long-term control of lymphoedema, identified only three controlled trials for inclusion. These randomised a total of 150 adults to different levels of physical treatment. One trial involved 42 women with unilateral lymphoedema of the upper limb following treatment for breast cancer. One group received eight sessions of MLD in two weeks and training in self-massage and both this group and the control group wore flat-knit compression sleeves. The reductions in excess arm volume and symptoms were similar in the two groups.

A second trial involved 25 women from a local follow-up breast clinic. They were trained in self-administered massage and randomised to wear an elastic compression sleeve or no additional treatment. The dropout rate was high, particularly in the control group, although the authors concluded that wearing a compression sleeve was beneficial. The third trial involved 83 mostly female participants from a lymphoedema clinic. Around two thirds had upper limb oedema. They were all taught self-administered massage. One group received a 19-day bandaging course before being fitted with hosiery. The other group wore hosiery from the start of the trial. The reduction in excess limb volume was consistently greater in those who started with multi-layer bandaging.

All three trials had methodological limitations, and as their data could not be combined, and they recruited only small numbers of participants, questions relating to the effect of this type of treatment could not be answered by this review.

\section{B A C K G R O U N D}

Lymphoedema is a chronic and progressive condition resulting from an abnormality of, or damage to the lymphatic system. Any reduction in the capacity of the lymphatic system to drain fluid from the interstitium and return it to the blood circulation will cause fluid to build up in the skin and subcutaneous tissues of the affected part of the body (Levick 1991; Mortimer 1995).

Lymphoedema has many causes but the main ones are: 
- Cancer and its treatment - leading to secondary lymphoedema;

- Congenital abnormalities of the lymphatic system - so called primary lymphoedema;

- Chronic Venous Disease of the lower limb - lympho-venous oedema;

- Filariasis, a parasitic infection - leading to secondary lymphoedema

The incidence of lymphoedema following breast cancer treatment is difficult to assess due to differences in assessments of diagnosis, measurement and follow up time. Petrek 1998 and Erickson 2001 conducted systematic reviews to assess the incidence of breast cancer lymphoedema. The included studies assessed the incidence in Europe, Australia and North America. Using different search strategies Petrek 1998 found eight studies which included incidence and Erickson 2001 found ten including potentially two studies which were too recent to include in the review by Petrek 1998. Four papers were included in both reviews although one paper was judged to be retrospective by Petrek 1998 and prospective by Erickson 2001. Three methods of assessing lymphoedema were included; volume, limb circumference and self-report. The time scale used for follow up also varies. Petrek 1998 report incidence figures of 6 to $30 \%$ however the table used to explain these figures is unclear. Only papers which covered axillary dissection were used in the review by Erickson 2001 and a range of 2.4 to $56 \%$ is found. The range in follow up, if details were available at all, seems to result in this wide variety in findings. The figures for lymphoedema of the lower limb are even less reliable but it appears to be a major problem. In many other cancers (for example melanoma, soft tissue sarcoma and pelvic tumours) the treatment often compromises lymphatic drainage routes; chronic venous insufficiency is also a major contributory factor in the development of chronic lower limb oedema, as is filariasis, a parasitic infection endemic in parts of India and Africa. Moffatt 2003 surveyed healthcare providers in South West London and found a crude prevalence of lymphoedema from any cause of 1.33 per 100,000 . The incidence of arm oedema was much higher in women, which reflects the large number of women developing lymphoedema following the development of breast cancer.

Lymphoedema can result in significant physical and psychological morbidity. Swelling causes a disproportion in the size of a part of the body and as such can interfere with mobility and affect the sufferers' perceptions of themselves (Tobin 1993). In addition to an increase in size, the affected subcutaneous tissues gradually thicken and fibrose forming a solid component to the swelling (Foldi 1985; Mortimer 1995). Pain and discomfort feature among the physical problems associated with lymphoedema (Badger 1988; Carroll 1992), as do recurrent attacks of infection/inflammation ( Mortimer 1995); the latter are a result of reduced local immunity in the affected part of the body. The shape of the limb can become distorted (Badger 1997). The impact of these physical and psychosocial difficulties on the patients' quality of life has attracted little research interest. As things stand, the pre-morbid state has not been identified so that the prevention of lymphoedema is not yet an option.

The management of this condition involves decongesting the reduced lymphatic pathways in order to reduce the size of the limb; encouraging the development of collateral drainage routes and stimulating the function of remaining patent routes so as to control the swelling long-term (Foldi 1985; Mortimer 1995).

Traditionally, treatment in continental Europe has followed a "two-phase" approach. In the first phase a collection of different physical treatments are employed simultaneously for a course of daily treatment; these usually include:

- Multi-layer bandaging

- Care of the skin

- Manual lymph drainage (MLD)

- Exercises to promote lymph drainage.

- Pneumatic compression (is sometimes also included here).

This phase aims to reduce the size of the limb, reverse any distortion in the shape of the limb and any hardening of the subcutaneous tissues and improve the health of the skin.

In the second phase of treatment patients are usually required to:

- Wear strong compression hosiery to maintain the reduction in swelling;

- Carry out regular daily exercise;

- Have regular MLD, where possible.

The aim here is to maintain the improvements gained in the first phase.

There is a great deal of debate as to which components of a physical treatment programme are the most crucial and whether bandages are more effective at reducing oedema than compression hosiery. Outside continental Europe the lack of experienced therapists and inadequate resources mean that standard treatment is likely to consist of compression hosiery with advice on skin care and exercise. Where MLD is not available, patients are often taught to perform a simplified form known as "simple lymph drainage" (SLD) or "self-administered massage" (SAM) however as to which of these methods is the more effective, and what effect they have is the subject of much speculation by therapists. Pneumatic compression therapy (PCT) used both as a way of reducing oedema and 
of controlling it, is an approach on which opinion is also divided. While PCT has been demonstrated to reduce swelling, the way in which it does so and the possibility of the rapid displacement of fluid to elsewhere in the body have caused concern. PCT does not obviate the need to contain the limb with hosiery and it is not clear that it provides anything which movement, whilst wearing a short-stretch bandage or compression hosiery, does not also provide. The role of weight control and regular exercises in the management of lymphoedema is also thought to be important.

\section{O B J E C T I VES}

1. To assess the effect of physical treatment programmes on the volume, shape, condition and long-term (six months) control of oedema in lymphoedematous limbs.

2. To assess the psycho-social benefits of physical treatment.

\section{METHODS}

\section{Criteria for considering studies for this review}

\section{Types of studies}

Only randomised controlled trials were included in this review. If insufficient details were provided as to the randomisation procedure, authors were contacted for more details. However, due to the lack of detail obtained in relation to the randomisation method, those trials described as randomised were included in the review.

\section{Types of participants}

Studies had to recruit adults (greater than 18 years of age) with a diagnosis of lymphoedema defined as clinically detectable oedema from a peripheral cause of greater than three months duration. Follow up had to be for at least six months. In patients with unilateral oedema, the increase in limb volume of the swollen limb had to be at least $10 \%$ above that of the contra-lateral normal limb volume. This cut-off point was based on information regarding the per cent difference in volume between left and right limbs in the normal population, which can be as high as 8 to $9 \%$ (Sitzia 1997). All types of lymphoedema were included, non cancer-related and cancer- related. Cancer patients had to have completed their cancer treatment at least six months before entering the trial and could not have evidence of recurrent malignant disease when going into the trial. If multi-layer bandaging was the intervention being studied then the participants could not have already received a course of treatment involving bandaging in the six months preceding their entry into the trial.

\section{Types of interventions}

- Multi-layer bandaging compared to hosiery

- Hosiery/multi-layer bandaging compared to exercise/no treatment

- Hosiery/multi-layer bandaging compared to MLD/

SLD/SAM

- MLD compared to SLD/SAM

- MLD/SLD/SAM compared to exercise/no treatment

- Exercise compared to no treatment

- PCT compared to hosiery/Multi-layer bandaging/SLD/

SAM/exercise

- PCT compared to no treatment

\section{Types of outcome measures}

Main outcomes

1. Volume measurement of limbs - reports of circumference measurements alone were not sufficient unless taken at $4 \mathrm{~cm}$ intervals when they could be converted to volume measurements by the formula circumference squared over Pi. Volume could be measured by:

- water displacement;

- electronic volumeter;

- calculated from surface measurements.

2. Where lymphoedema was unilateral the normal limb should act as the patient's own control - volume had to be expressed as the excess limb volume over the normal limb volume; any reduction/ increase should be reported as the percentage reduction/increase in the excess limb volume.

3. Impact on quality of life.

4. Impact on patient's sense of well-being.

5. Impact on patient's mobility.

6. Reduction in recurrent infections.

Any psychological benefit such as changes in a sense of well-being/ quality of life or improvement in body image had to be reported using validated scales. Other aspects of physical morbidity such as an improvement in the condition of the skin and the quality of the tissues in the limb, improvement in the shape of the limb and increased mobility had to be assessed objectively whenever possible. Long-term and short- term adverse effects related to the interventions were noted.

\section{Search methods for identification of studies}

The Cochrane Breast Cancer Specialised Register contains the results of searching a wide list of databases together with handsearching of specialised journals and conference proceedings. For this updated version of the review, the authors decided not to continue to search some databases previously searched, as the trials register is likely to contain all relevant trials. However, supplementary 
searching for this updated version of the review was undertaken of the larger electronic databases separately by the authors as an additional check.

\section{Databases searched for this current updated review:}

- Cochrane Breast Cancer Specialised Register

For the first full version of this review (New Reference), the Specialised Register maintained by the Cochrane Breast Cancer Group was searched in September 2003 (details of search strategies used by the group for the identification of studies and the procedure used to code references are outlined in the group's module http:// www.mrw.interscience.wiley.com/cochrane/clabout/articles/ BREASTCA/frame.html). Studies coded as 'lymphoedema' on the Specialised Register were extracted for consideration. This search was repeated in October 2007 for this update.

- Cochrane Central Register of Controlled Trials (Issue 1, 2008)

See Appendix 1

- CINAHL (1982 to February 2008)

See Appendix 2

- MEDLINE (1950 to February 2008)

See Appendix 3

- EMBASE (1980 to February 2008)

See Appendix 4

- National Research Register (NRR) (February 2008)

Potentially relevant studies from this search were extracted for consideration and attempts made to locate the authors.

See Appendix 5

Databases searched for original review only (Badger C, 2004):

- BNI (September 2003)

- CANCERLIT (September 2003)

- British Library Index (September 2003)

- UnCover (September 2003) ("UnCover Title and Subject

Lists" is a database listing journal contents pages)

- PASCAL (September 2003)

Search terms used for the above databases are included in Appendix 6. Searches went back, where possible, as far as the 1960s. In order to identify the "grey literature" SIGLE was also searched.

Other sources searched for original review only (Badger C, 2004):

- International Society of Lymphology biennial congress proceedings (hand searched September 2003)

- International experts in the field were contacted to see if they held any unpublished data, as were those found to be presenting relevant papers in conference proceedings.
All 353 members of the International Society of Lymphology were contacted by letter to ask if they had any results of past or ongoing studies that could be considered for inclusion in the review. Only 30 replies were received and none of these uncovered data that could be included in this review.

\section{Data collection and analysis}

\section{Selection}

One reviewer scanned the titles and abstracts of the papers found through searches (CB). Those clearly not relevant based on reading of the abstract (for example if it was clear that they did not describe studies, or did not relate to lymphoedema) were excluded; if no abstract was available or if it was not immediately clear that the paper was not relevant then the full publication was retrieved for closer review. A second reviewer (NP) looked at a sample of those discarded to check that nothing relevant had been lost. This process was independently undertaken by two reviewers in the 2008 update (AL and JB) and differences resolved by consensus.

Two reviewers (CB and NP or AL and JB) independently read all the retrieved papers under consideration and independently assessed their eligibility for inclusion according to the criteria set out for trials above. An eligibility form was designed to aid the selection of papers and piloted before use. A third reviewer was designated to resolve any disagreement over the inclusion of any particular trial.

\section{Data extraction}

We designed and piloted a data extraction form before use on the selected papers. Data extraction was duplicated by the second reviewer (NP) and any disagreements resolved by the third reviewer (KS). Wherever possible the following data were extracted from the selected studies:

1. Details of participants including demographic characteristics, source of recruitment, site of oedema, cause of oedema, duration of oedema, relevant co-existing medical conditions, and details of the subjects' occupations.

2. Where relevant, details of the type and treatment of any cancer, including the patient's cancer status at the time of the trial.

3. Details of the experimental and control interventions, including the length of time they were applied for and confirmation that CDT had not been used in the 6 months before the trial.

4. Details relating to the homogeneity of the two treatment groups, e.g. the severity of oedema at the start of the trial and/or the duration of oedema.

5. The methods of assessment of limb volume and other relevant outcomes.

6. Details of any financial support that might introduce a conflict of interest;

7. The numbers of participants allocated to each group and the numbers lost to follow-up or excluded, together with the reasons why.

Analysis 
None of the studies identified compared the same intervention, therefore data were not combined. We did not perform sub-group analysis due to insufficient data. Analysis was by intention to treat. As no studies were found comparing the same interventions, the results of each study have been described in text form. Further statistical analysis was not possible.

There were insufficient data to retrieve information on infection and quality of life.

\section{R E S U L T S}

\section{Description of studies}

See: Characteristics of included studies; Characteristics of excluded studies; Characteristics of ongoing studies.

Search results

As a result of the 2003 search, we identified 195 papers for possible inclusion. Of these, 185 were found to be ineligible on the basis that they were not randomised controlled trials. Of the remaining 10 studies, all were described as randomised. For one study (Thiadens 1999) only the abstract was available and to date the review authors have been unable to obtain the full report; the design of a trial reported in another paper (Bergan 1998) remains uncertain and awaits clarification. These are listed in the Studies Awaiting Assessment Table. Of the remaining eight studies, data were only extracted from only three as the other five did not meet the inclusion criteria. Reasons for their exclusion are summarised in the Characteristics of Excluded Studies Table.

The 2006 search of the specialised register identified a further possible 33 studies and the 2007 search identified a further possible 21 studies. Of these, eight studies were retrieved as potentially eligible RCTs and read in full by two reviewers (AL and SE). Two studies are ongoing (Didem 2008 (this is longer follow up of one of the publications excluded because of short follow up); Schmitz 2006) and are listed in the Ongoing Studies Table. Seven studies did not fulfill the eligibility criteria for the review and are listed in the Excluded Studies Table. The 2008 search of other electronic databases identified a further possible 477 studies. One study was retrieved for further inspection but was excluded as it did not meet the eligibility criteria (Irdesel 2007).

Most of the potentially relevant studies were excluded because follow up was less than 6 months. These short term trials included 2 studies of pneumatic compression (Dini 1998; Johansson 1998), five studies comparing MLD with various types of control (Sitzia 2002; Williams 2002; Didem 2005; Wilburn 2006; McNeely 2004), one study of compression plus exercise vs exercise alone ( Johansson 2005) and one study of upper body exercise (McKenzie 2003). Another study had six month follow up but most participants continued with the experimental intervention, pneumatic compression, after 2 months follow up (Szuba 2002). Two studies were excluded because limb size was not assessed using the volume measurement (Bertelli 1991; Irdesel 2007) and one study was excluded because only a proportion of the participants (29\%) had lymphedema at baseline (Ahmed 2006). Full details of all of the excluded studies are found in Table 1.

Table 1. Details of excluded trials

\begin{tabular}{|c|c|c|c|c|c|}
\hline Study ID & Methods & Participants & Interventions & Outcomes & Notes \\
\hline $\begin{array}{l}\text { Ahmed } 2006 \\
\text { USA }\end{array}$ & $\begin{array}{l}6 \text { month parallel } \\
\text { group trial } \\
\text { Blocked randomisa- } \\
\text { tion procedure with } \\
\text { random number ta- } \\
\text { ble and stratifying } \\
\text { according to age and } \\
\text { body fat } \\
\text { 2 groups: } \\
\text { (1) weight training } \\
\text { twice a week ( } \mathrm{n}=23 \text { ) } \\
\text { (2) control } \\
\text { - no weight training } \\
\text { ( } \mathrm{n}=23 \text { ) }\end{array}$ & $\begin{array}{l}\text { Recruitment } \\
\text { was between Octo- } \\
\text { ber } 2001 \text { and June } \\
2002 . \\
\text { Breast cancer sur- } \\
\text { vivors living in the } \\
\text { greater Minneapo- } \\
\text { lis-Saint Paul area. } \\
\text { All appear to have } \\
\text { had axillary node } \\
\text { dissection. } \\
\text { Only a proportion } \\
\text { of the group had } \\
\text { lymphoedema }\end{array}$ & $\begin{array}{l}\text { (1) Weight training } \\
\text { twice a week for } 6 \\
\text { months for approx } 1 \\
\text { hour } \\
\text { (2) Control - not } \\
\text { clearly described. It } \\
\text { appears they had no } \\
\text { weight training. }\end{array}$ & $\begin{array}{l}\text { - Change in arm cir- } \\
\text { cumference } \\
\text { - Incidence } \\
\text { of lymphoedema at } \\
6 \text { months (measured } \\
\text { by arm circumfer- } \\
\text { ence or self report) }\end{array}$ & $\begin{array}{l}\text { Only a proportion } \\
\text { of the participants } \\
\text { had lymphoedema } \\
\text { at baseline (13\%). } \\
\text { Trial does not use } \\
\text { limb volume as the } \\
\text { method of assessing } \\
\text { change in size. }\end{array}$ \\
\hline
\end{tabular}


Table 1. Details of excluded trials (Continued)

\begin{tabular}{|c|c|c|c|c|c|}
\hline & $\begin{array}{l}\text { Withdrawn/ } \\
\text { excluded: } \\
\text { Not clear. It appears } \\
\text { as though the anal- } \\
\text { yses were only un- } \\
\text { dertaken on women } \\
\text { who had axillary } \\
\text { dissection but the } \\
\text { numbers vary from } \\
22 \text { to } 23 \text { in control } \\
\text { group. Dropouts } \\
\text { from original trial: } \\
\text { (1) } 4 / 42 \text { (10\%) - } \\
2 \text { breast cancer re- } \\
\text { currence, } 2 \text { personal } \\
\text { reasons; (2) 3/43 } \\
7 \% \text { - } 2 \text { breast cancer } \\
\text { recurrence, } 1 \text { per- } \\
\text { sonal reasons }\end{array}$ & $\begin{array}{l}\text { (measured by arm } \\
\text { circumference mea- } \\
\text { surements, self re- } \\
\text { port of diagnosis } \\
\text { and self report of } \\
\text { symptoms) } \\
\text { (1) } \\
\text { Age: } 52.3 \text { (7.7) yrs } \\
\text { Self report of lymph } \\
\text { diagnosis: } \\
\text { (30.4\%) } \\
\text { Self report of lymph } \\
\text { symptoms: } \\
\text { (43.4\%) } \\
\text { Lymph by arm circ: } \\
4 \text { (17.4\%) } \\
\text { Time since br ca di- } \\
\text { agnosis: } 22.3 \text { ( } 7 \text { to } \\
43) \text { mths } \\
\text { (2) } \\
\text { Age: } 51.7 \text { (7.5) yrs } \\
\text { Self report of lymph } \\
\text { diagnosis: } \\
(26.1 \%) \\
\text { Self report } \quad 6 \\
\text { lymph symptoms: } 7 \\
(30.4 \%) \\
\text { Lymph by arm circ: } \\
4(17.4 \%) \\
\text { Time since br ca di- } \\
\text { agnosis: } 21.9 \text { ( } 11 \text { to } \\
57) \text { mths }\end{array}$ & & & \\
\hline $\begin{array}{l}\text { Bertelli } 1991 \\
\text { Italy }\end{array}$ & $\begin{array}{l}6 \text { month parallel } \\
\text { group trial } \\
\text { Method } \\
\text { randomisation not } \\
\text { stated. } \\
\text { Two groups: } \\
\text { Group 1: Hosiery } \\
\text { alone ( } \mathrm{n}=37) \\
\text { Group 2: Hosiery + } \\
\text { electrically } \\
\text { stimulated drainage } \\
\text { (ESD) }(\mathrm{n}=37)\end{array}$ & $\begin{array}{l}\text { All had unilateral } \\
\text { oedema of the up- } \\
\text { per limb following } \\
\text { trt for breast ca. } \\
\text { All had axillary node } \\
\text { dissection. } \\
\text { All had Delta value } \\
>10 \mathrm{cms}<20 \mathrm{cms} \\
\text { Group } 1 \text { : } \\
\text { Median (range) } \\
\text { Age }=64(45-77)\end{array}$ & $\begin{array}{l}\text { Group 1: } \\
\text { Compression sleeve } \\
\text { worn for } 6 \text { hours a } \\
\text { day } \\
\text { Group 2: } \\
\text { Compression sleeve } \\
\text { worn for } 6 \text { hours a } \\
\text { day } \\
\text { ESD 10x30 min } \\
\text { over } 2 \text { weeks; gap of } \\
5 \text { weeks then same } \\
\text { cycle repeated. }\end{array}$ & $\begin{array}{l}\text { - Change in size es- } \\
\text { timated by taking } \\
\text { circumference mea- } \\
\text { sure- } \\
\text { ments at } 7 \text { points } \\
\text { along both swollen } \\
\text { \& normal limb, es- } \\
\text { tablishing the differ- } \\
\text { ence between them, } \\
\text { and totalling these. } \\
\text { the sum of the dif- } \\
\text { ferences be- } \\
\text { tween swollen and } \\
\text { normal were desig- }\end{array}$ & $\begin{array}{l}\text { Trial is long enough } \\
\text { but does not use } \\
\text { limb volume as the } \\
\text { method of assessing } \\
\text { change in size. }\end{array}$ \\
\hline
\end{tabular}


Table 1. Details of excluded trials (Continued)

\begin{tabular}{|c|c|c|c|c|c|}
\hline & $\begin{array}{l}\text { Withdrawn/ } \\
\text { excluded: } \\
\text { 14/74 } \\
\text { Group 1: 6/37 } \\
2 \text { refused treatment } \\
4 \text { lost to follow-up } \\
\text { Group 2: } 8 / 37 \\
2 \text { refused treatment } \\
1 \text { had lymphangitis; } \\
2 \text { lost to follow-up; } \\
3 \text { withdrawn be- } \\
\text { cause oedema wors- } \\
\text { ened. }\end{array}$ & $\begin{array}{l}\text { No. }(\%) \\
\text { Radical mast = } 17 \\
(46) \\
\text { Mod. radical mast = } \\
12(32.4) \\
\text { Quandrantectomy } \\
=8(21.6) \\
\text { Radiotherapy = } 14 \\
(37.8) \\
\text { Chemotherapy = } 10 \\
(27) \\
\text { Prev. } \\
\text { trt for l'oedema = } 19 \\
(51.3) \\
\text { Prev. lymphangitis = } \\
12(32.4) \\
\text { Group } 2: \\
\text { Median (range) } \\
\text { age = 64 (48-78) } \\
\text { No. }(\%) \\
\text { Radical mast = } 10 \\
(27) \\
\text { Mod. radical mast = } \\
18(48.7) \\
\text { Quandrantectomy } \\
=9(24.3) \\
\text { Radiotherapy = } 14 \\
(37.8) \\
\text { Chemotherapy = } 6 \\
(16.2) \\
\text { Prev. } \\
\text { trt for l'oedema = } 16 \\
(43.2) \\
\text { Prev. lymphangitis = } \\
12(32.4)\end{array}$ & $\begin{array}{l}\text { Both groups re- } \\
\text { ceived advice of skin } \\
\text { care and prevention } \\
\text { of infection. }\end{array}$ & $\begin{array}{l}\text { nated "Delta". } \\
\text { Response was deter- } \\
\text { mined by the } \% \\
\text { change in the fi- } \\
\text { nal Delta value com- } \\
\text { pared to baseline. }\end{array}$ & \\
\hline $\begin{array}{l}\text { Didem } 2005 \\
\text { Turkey }\end{array}$ & $\begin{array}{l}4 \text { week trial. } \\
\text { Randomi- } \\
\text { sation by unmarked } \\
\text { envelopes. } \\
2 \text { groups: } \\
\text { (1) Complex decon- } \\
\text { gestive physiother- } \\
\text { apy (CDP) (n=27) } \\
\text { (2) Standard phys- } \\
\text { iotherapy (SP) }\end{array}$ & $\begin{array}{l}\text { All had developed } \\
\text { lymphoedema after } \\
\text { the first year from } \\
\text { surgery (average du- } \\
\text { ration of lymph at } \\
\text { time of treatment } \\
\text { was } 3 \text { years). } \\
60 \% \text { had moder- } \\
\text { ate lymph; } 40 \% \text { had } \\
\text { mild } \\
\text { lymph (but this var- }\end{array}$ & $\begin{array}{l}\text { Group 1: } \\
\text { CDP: lymph } \\
\text { drainage, } \\
\text { multilayer compres- } \\
\text { sion bandage, eleva- } \\
\text { tion, remedial exer- } \\
\text { cises and skin care } \\
\text { Group 2: } \\
\text { SP: bandage, eleva- } \\
\text { tion, head-neck and } \\
\text { shoulder exercises }\end{array}$ & $\begin{array}{l}\text { - Edema of the arm } \\
\text { (assessed by circum- } \\
\text { ference } \\
\text { measurements) } \\
\text { - Volume of the arm } \\
\text { (assessed with water } \\
\text { displacement) } \\
\text { - Shoulder mobility } \\
\text { (assessed by exten- } \\
\text { sion-flexion, abduc- } \\
\text { tion-adduction, ex- } \\
\text { ternal rotation) }\end{array}$ & $\begin{array}{l}\text { Trial does not fol- } \\
\text { low the patients for } \\
\text { long enough. The } \\
\text { authors state that } \\
\text { they plan to report } \\
\text { on long term fol- } \\
\text { low up ( } 6 \text { months, } \\
12 \text { months and } 24 \\
\text { months). The au- } \\
\text { thors were } \\
\text { contacted but have } \\
\text { not submitted their }\end{array}$ \\
\hline
\end{tabular}


Table 1. Details of excluded trials (Continued)

\begin{tabular}{|c|c|c|c|c|c|}
\hline & $\begin{array}{l}(\mathrm{n}=26) \\
\text { Withdrawn/ } \\
\text { excluded: } \\
\text { There appear to be } \\
\text { no withdrawals af- } \\
\text { ter randomisation. } \\
\text { Prior to randomi- } \\
\text { sation: } 1 / 28 \text { from } \\
\text { group } 1 \text { withdrew } \\
\text { because of br ca re- } \\
\text { currence; } 2 / 28 \text { with- } \\
\text { drew from group } 2 \\
\text { because of arm in- } \\
\text { fection. }\end{array}$ & $\begin{array}{l}\text { ied by randomised } \\
\text { group (there are er- } \\
\text { rors in the publica- } \\
\text { tion). } \\
\text { All patients had un- } \\
\text { dergone axillary dis- } \\
\text { section with a range } \\
\text { of } 2 \text { - } 35 \text { nodes re- } \\
\text { moved. } \\
\text { (1) } \\
\text { Mild lymph: } 7.4 \% \\
\text { Moderate lymph: } \\
92.3 \% \\
\text { Mild: age } 53.1(3.1) \\
\text { yrs } \\
\text { Moderate: age } 61.3 \\
\text { (7.2) yrs } \\
\text { Mild: weight } 66.3 \\
\text { (9.8) kg } \\
\text { Moderate: weight } \\
73.9 \text { (13.2) kg } \\
\text { Mild: history of cel- } \\
\text { lulites } 8.3 \% \\
\text { Moderate: history } \\
\text { of cellulites } 20 \% \\
\text { (2) } \\
\text { Mild lymph: } 73.1 \% \\
\text { Moderate lymph: } \\
26.9 \% \\
\text { Mild: age } 54.7 \\
\text { (12.1) yrs } \\
\text { Moderate: age } 63.6 \\
\text { (0.7) yrs } \\
\text { Mild: weight } 64.7 \\
\text { (11.4) kg } \\
\text { Moderate: weight } \\
71.5 \text { (6.8) kg } \\
\text { Mild: history of cel- } \\
\text { lulites } 0 \% \\
\text { Moderate: history } \\
\text { of cellulites } 29.4 \%\end{array}$ & $\begin{array}{l}\text { and skin care } \\
\text { All patients had } \\
\text { their physiotherapy } \\
\text { once a day, } 3 \text { days } \\
\text { a week for } 4 \text { weeks } \\
\text { and they were blind } \\
\text { to the intervention. }\end{array}$ & & $\begin{array}{l}\text { longer term follow } \\
\text { up. }\end{array}$ \\
\hline $\begin{array}{l}\text { Dini } 1998 \\
\text { Italy }\end{array}$ & $\begin{array}{l}9 \text { week parallel } \\
\text { group trial } \\
\text { Randomisation } \\
\text { achieved by phone }\end{array}$ & $\begin{array}{l}\text { All parti- } \\
\text { ciants had unilateral } \\
\text { upper limb oedema } \\
\text { following "radical" } \\
\text { breast surgery + axil- }\end{array}$ & $\begin{array}{l}\text { Group 1: } \\
\text { Pneu- } \\
\text { matic compression } \\
(60 \mathrm{mmHg}) 2 \mathrm{hr} \text { ses- }\end{array}$ & $\begin{array}{l}\text { - Change in size es- } \\
\text { timated by taking } \\
\text { circumference mea- } \\
\text { sure- }\end{array}$ & $\begin{array}{l}\text { Trial does not follow } \\
\text { patients up for long } \\
\text { enough (i.e. at least } \\
6 \text { months). }\end{array}$ \\
\hline
\end{tabular}

Physical therapies for reducing and controlling lymphoedema of the limbs (Review)

Copyright (? 2008 The Cochrane Collaboration. Published by John Wiley \& Sons, Ltd. 
Table 1. Details of excluded trials (Continued)

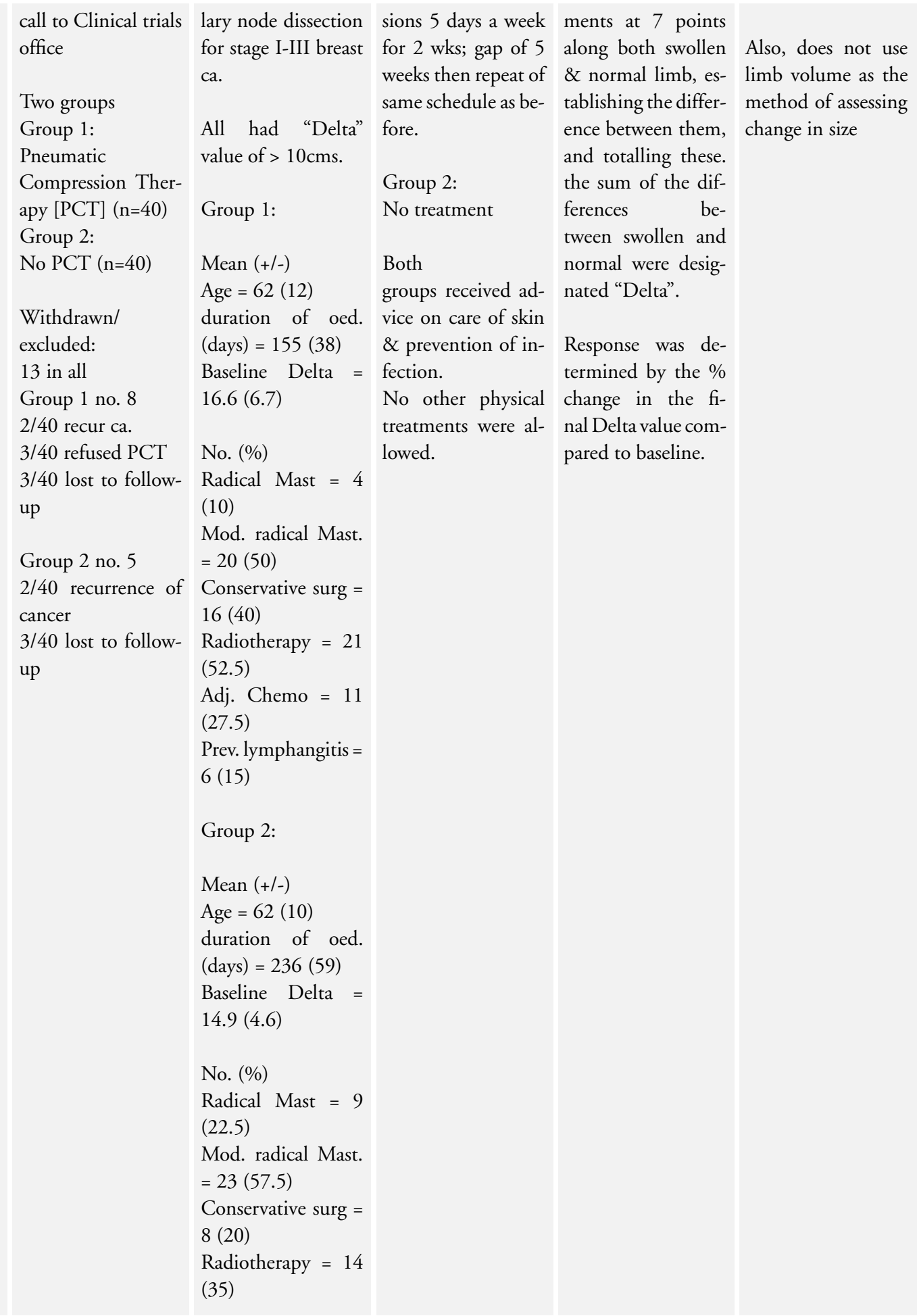


Table 1. Details of excluded trials (Continued)

\begin{tabular}{|c|c|c|c|c|c|}
\hline & & $\begin{array}{l}\text { Adj. Chemo }=8 \\
(20) \\
\text { Prev. lymphangitis }= \\
10(25)\end{array}$ & & & \\
\hline $\begin{array}{l}\text { Irdesel } 2007 \\
\text { Turkey }\end{array}$ & $\begin{array}{l}6 \text { month parallel } \\
\text { group trial } \\
\text { Ran- } \\
\text { domisation method } \\
\text { not clear from ab- } \\
\text { stract } \\
2 \text { groups: } \\
\text { (1) } \\
\text { ercises and compres- } \\
\text { sion garment, n=10 } \\
\text { (2) exercises alone, } \\
\mathrm{n}=9 \\
\text { Withdrawn/ } \\
\text { excluded: Not clear } \\
\text { from abstract }\end{array}$ & $\begin{array}{l}\text { Details not clear } \\
\text { from abstract }\end{array}$ & $\begin{array}{l}\text { Group 1: } \\
\text { Exercises and com- } \\
\text { pression garment } \\
\text { Group 2: } \\
\text { Exercises alone }\end{array}$ & $\begin{array}{l}\text { - Reduction in lym- } \\
\text { phoedema volume } \\
\text { (assessed by mea- } \\
\text { surement of arm cir- } \\
\text { cumference) } \\
\text { - Improvement in } \\
\text { shoulder range of } \\
\text { motion } \\
\text { - Symptoms such } \\
\text { as pain and tender } \\
\text { points }\end{array}$ & $\begin{array}{l}\text { Trial does not use } \\
\text { limb volume as the } \\
\text { method of assessing } \\
\text { change in size }\end{array}$ \\
\hline $\begin{array}{l}\text { Johansson } 1998 \\
\text { Sweden }\end{array}$ & $\begin{array}{l}4 \\
\text { week parallel group } \\
\text { trial in } 2 \text { parts } \\
\text { Part I all subjects } \\
\text { received same treat- } \\
\text { ment } \\
\text { Part II randomised } \\
\text { to } 2 \text { different inter- } \\
\text { ventions } \\
\text { Method } \\
\text { randomisation not } \\
\text { stated } \\
\text { 2 groups } \\
\text { Group 1: } \\
\text { Manual Lymph } \\
\text { Drainage [MLD] } \\
\text { (n=14) } \\
\text { Group 2: } \\
\text { Sequential Pneu- } \\
\text { matic Compression } \\
\text { [SPC] (n=14) } \\
\text { Withdrawn/ }\end{array}$ & $\begin{array}{l}\text { Recruitment } \\
\text { was over a } 2.5 \text { year } \\
\text { period. } \\
\text { Patients attend- } \\
\text { ing Dept. of phys- } \\
\text { ical therapy with } \\
\text { unilateral breast ca- } \\
\text { related upper limb } \\
\text { lymphoedema. } \\
\text { All had axillary node } \\
\text { dissection. } \\
\text { Lymphoedema de- } \\
\text { fined as excess limb } \\
\text { vol >10\% } \\
\text { Group 1: } \\
\text { Median (q1-q3) } \\
\text { Age = } 64 \text { (52.5- } \\
69.5) \\
\text { Duration of oedema } \\
\text { (months) } 14.0 \text { (3.0- } \\
76.5)\end{array}$ & $\begin{array}{l}\text { All participants be- } \\
\text { gan with } 2 \text { weeks of } \\
\text { wearing a compres- } \\
\text { sion sleeve } \\
\text { 3rd week } \\
\text { Group 1: } \\
\text { Received MLD for } \\
45 \text { mins/day; } 5 / 7 \text { for } \\
2 \text { weeks at same time } \\
\text { each day } \\
\text { Group } 2 \text { : } \\
\text { Received sequen- } \\
\text { tial pneumatic com- } \\
\text { pression therapy } \\
\text { with the Lympha- } \\
\text { Press } 2 \text { hrs/day ( } 40- \\
60 \text { mmHg) } \\
\text { Both groups contin- } \\
\text { ued to wear a com- } \\
\text { pression sleeve. }\end{array}$ & $\begin{array}{l}\text { - \% reduction in ex- } \\
\text { cess limb volume } \\
\text { measured by water } \\
\text { displacement } \\
\text { - Body weight } \\
\text { - Passive mobility of } \\
\text { shoulder measured } \\
\text { by goniometer } \\
\text { - Isometric muscle } \\
\text { strength measured } \\
\text { by dynamometer } \\
\text { - Patient's subjective } \\
\text { assessment of: pain, } \\
\text { heaviness, tension, } \\
\text { function, paresthe- } \\
\text { sia. }\end{array}$ & $\begin{array}{l}\text { Trial does not follow } \\
\text { patients up for long } \\
\text { enough (i.e. at least } \\
6 \text { months) }\end{array}$ \\
\hline
\end{tabular}


Table 1. Details of excluded trials (Continued)

\begin{tabular}{|c|c|c|c|c|c|}
\hline & $\begin{array}{l}\text { excluded } \\
\text { End of Part } 1-1 / 28 \\
\text { because oedema re- } \\
\text { solved } \\
\text { Part } 2 \text { - } 4 \text { in all, } 2 \\
\text { from each group. } \\
2 \text { patients had recur- } \\
\text { rent cancer; } \\
1 \text { patient had } \\
\text { erysipelas; } \\
1 \text { patient could not } \\
\text { attend for follow-up }\end{array}$ & $\begin{array}{l}\text { No. } \\
\text { Partial mastectomy } \\
=1 \\
\text { Mastectomy = } 11 \\
\text { Radiotherapy = } 10 \\
\text { Group 2: } \\
\text { Median (q1-q3) } \\
\text { Age = 57.5 (47.5- } \\
69.5) \\
\text { Duration of oedema } \\
\text { (months) } 6.0 \quad(2.3- \\
68.3) \\
\text { No. } \\
\text { Partial mastectomy } \\
=2 \\
\text { Mastectomy = } 10 \\
\text { Radiotherapy = } 8\end{array}$ & & & \\
\hline $\begin{array}{l}\text { Johansson } 2005 \\
\text { Sweden }\end{array}$ & $\begin{array}{l}5 \text { day crossover trial. } \\
\text { Randomisation } \\
\text { method not stated. } \\
2 \text { groups: } \\
\text { (1) } \\
\text { arm exercises with } \\
\text { compression sleeve } \\
\text { ( } \mathrm{n}=16 \text { first) } \\
(2) \text { arm exercises } \\
\text { without } \\
\text { compression sleeve } \\
\text { ( } \mathrm{n}=15 \text { first) } \\
\text { Withdrawn/ } \\
\text { excluded: } \\
4 / 42 \text { withdrew be- } \\
\text { cause of personal } \\
\text { reasons; } 7 / 42 \text { did } \\
\text { not reach the eligi- } \\
\text { bility criteria of at } \\
\text { least a } 10 \% \text { greater } \\
\text { arm volume in the } \\
\text { affected arm (after } \\
\text { randomisation) }\end{array}$ & $\begin{array}{l}\text { Recruitment was in } \\
\text { September } 2002 . \\
\text { Patients from the } \\
\text { physiothera- } \\
\text { pists registry of lym- } \\
\text { phoedema patients } \\
\text { at the Physiother- } \\
\text { apy Department of } \\
\text { Vaxjo Central Hos- } \\
\text { pital and the Lym- } \\
\text { phedema } \\
\text { Unit, Lund Univer- } \\
\text { sity Hospital, Lund } \\
\text { Sweden. } \\
\text { Lymphoedema de- } \\
\text { fined as } 10-40 \% \\
\text { greater arm volume } \\
\text { in affected arm. } \\
\text { Demographic char- } \\
\text { acteristics not given } \\
\text { separately by group. } \\
\text { Age: } 55.3 \text { (7.3) yrs } \\
\text { Surgery: } \\
\text { partial n=13; mas- }\end{array}$ & $\begin{array}{l}\text { All participants } \\
\text { wore a compression } \\
\text { sleeve for } 2 \text { weeks } \\
\text { before the trial. } \\
\text { Trial was over } 5 \\
\text { days: } \\
\text { Training exercise on } \\
\text { day } 1 \text {, assessments } \\
\text { before, immediately } \\
\text { after and } 24 \text { hours } \\
\text { after. } \\
\text { Then training exer- } \\
\text { cise on day } 3 \text { with } \\
\text { assessments before, } \\
\text { imme- } \\
\text { diately after and } 24 \\
\text { hours after. The par- } \\
\text { ticipants appear to } \\
\text { be crossed over to } \\
\text { the alternate treat- } \\
\text { ment for the } 2 \text { nd } \\
\text { training session. }\end{array}$ & $\begin{array}{l}\text { - Arm volume (mea- } \\
\text { sured by water dis- } \\
\text { placement method } \\
\text { and multiple fre- } \\
\text { quency bioelectrical } \\
\text { impedance analysis) } \\
\text { - Borg's scale for per- } \\
\text { ceived exertion }\end{array}$ & $\begin{array}{l}\text { Trial does not follow } \\
\text { patients up for long } \\
\text { enough (at least } 6 \\
\text { months) }\end{array}$ \\
\hline
\end{tabular}


Table 1. Details of excluded trials (Continued)

\begin{tabular}{|c|c|c|c|c|c|}
\hline & & $\begin{array}{l}\text { tectomy } \mathrm{n}=18 \\
\text { No of axillary nodes } \\
\text { dissected: } \quad 13.3 \\
\text { (5.2) } \\
\text { Duration of edema: } \\
66.7(51.7) \text { months } \\
\text { BMI: } 25.9(3.2)\end{array}$ & & & \\
\hline $\begin{array}{l}\text { McKenzie } 2003 \\
\text { Canada }\end{array}$ & $\begin{array}{l}8 \text { week parallel } \\
\text { group trial. } \\
\text { Randomisation } \\
\text { method not stated. } \\
2 \text { groups: } \\
\text { (1) Exercise, n=7 } \\
\text { (2) Control (no ex- } \\
\text { ercise), n=7 } \\
\text { Withdrawn/ } \\
\text { excluded: } \\
\text { There did not ap- } \\
\text { pear to be any with- } \\
\text { drawals from the } \\
\text { trial. }\end{array}$ & $\begin{array}{l}\text { Recruitment details } \\
\text { not provided. } \\
\text { Lymphoedema de- } \\
\text { fined } \\
\text { as unilateral and be- } \\
\text { tween } 2 \text { and } 8 \text { cm on } \\
\text { at least one measure- } \\
\text { ment point. } \\
\text { Group 1: } \\
\text { Age: } 56.4(10.4) \text { yrs } \\
\text { Weight: } 77.8 \text { (20.6) } \\
\text { kg } \\
\text { BMI: } 29.1 \text { (6.6) } \\
\text { Group 2: } \\
\text { Age: } 56.9(8.2) \text { yrs } \\
\text { Weight: } 67.3 \text { (9.1) } \\
\text { kg } \\
\text { BMI; } 25.6(3.3)\end{array}$ & $\begin{array}{l}\text { A compression } \\
\text { sleeve was used daily } \\
\text { by all participants. } \\
\text { (1) } 8 \text { week exer- } \\
\text { cise program (re- } \\
\text { sistance training } 3 \\
\text { times per week plus } \\
\text { aerobic exercise us- } \\
\text { ing a Monark Re- } \\
\text { hab Trainer arm er- } \\
\text { gometer) } \\
\text { (2) No specific exer- } \\
\text { cise instruction }\end{array}$ & $\begin{array}{l}\text { - Arm circumfer- } \\
\text { ence } \\
\text { - Arm volume (as- } \\
\text { sessed by water dis- } \\
\text { placement) } \\
\text { - SF36 }\end{array}$ & $\begin{array}{l}\text { Trial does not fol- } \\
\text { low patients for long } \\
\text { enough (at least } 6 \\
\text { months). }\end{array}$ \\
\hline $\begin{array}{l}\text { McNeely } 2004 \\
\text { Canada }\end{array}$ & $\begin{array}{l}4 \quad \text { week parallel } \\
\text { group trial. } \\
\text { Randomisation by } \\
\text { computer generated } \\
\text { code. } \\
2 \text { groups: } \\
\text { (1) MLD plus } \\
\text { multi-layered com- } \\
\text { pression bandaging, } \\
\text { n=25 } \\
\text { (2) Com- } \\
\text { pression bandaging } \\
\text { alone, n=25 } \\
\text { Withdrawn/ } \\
\text { excluded: } \\
5 / 50 \text { did not com- }\end{array}$ & $\begin{array}{l}\text { Recruit- } \\
\text { ment from Novem- } \\
\text { ber } 2000 \text { to De- } \\
\text { cember } 2001 \text { from } \\
\text { Cross Cancer Insti- } \\
\text { tute in Edmonton, } \\
\text { Canada. } \\
\text { Lymphoedema de- } \\
\text { fined as a mini- } \\
\text { mum of a } 150 \mathrm{ml} \\
\text { difference between } \\
\text { affected and unaf- } \\
\text { fected arms. } \\
\text { Group } 1 \text { : } \\
\text { Age: } 58 \text { (13) yrs } \\
\text { No lymph nodes re- } \\
\text { moved: } 12 \text { (6) }\end{array}$ & $\begin{array}{l}\text { All participants had } \\
\text { standard education } \\
\text { on proper arm and } \\
\text { skin care. } \\
\text { (1) } 45 \text { minutes of } \\
\text { daily MLD } 5 \text { times } \\
\text { per week (Vodder } \\
\text { method) plus short } \\
\text { stretch bandaging } \\
\text { (2) Short stretch } \\
\text { bandaging alone }\end{array}$ & $\begin{array}{l}\text { - Reduction in arm } \\
\text { lymphoedema vol- } \\
\text { ume (assessed by } \\
\text { water displacement } \\
\text { volumetry and arm } \\
\text { circumference) } \\
\text { This outcome was } \\
\text { assessed by indepen- } \\
\text { dent asses- } \\
\text { sors blinded to sub- } \\
\text { ject treatment as- } \\
\text { signment. }\end{array}$ & $\begin{array}{l}\text { Trial does not fol- } \\
\text { low patients for long } \\
\text { enough (at least } 6 \\
\text { months). }\end{array}$ \\
\hline
\end{tabular}


Table 1. Details of excluded trials (Continued)

\begin{tabular}{|c|c|c|c|c|c|}
\hline & $\begin{array}{l}\text { plete the study. } 2 \\
\text { withdrew because of } \\
\text { adverse events (1 } \\
\text { from group } 1 \text { be- } \\
\text { cause of a skin reac- } \\
\text { tion to bandaging, } \\
1 \text { from group } 2 \text { be- } \\
\text { cause of elbow dis- } \\
\text { comfort from } \\
\text { bandaging), } 3 \text { from } \\
\text { group } 2 \text { withdrew (1 } \\
\text { because of personal } \\
\text { reasons, } 2 \text { because of } \\
\text { dissatisfaction with } \\
\text { treatment response) } \\
.1 \text { additional partic- } \\
\text { ipant from group } 1 \\
\text { withdrew from the } \\
\text { assessment of arm } \\
\text { volume because of } \\
\text { an error in measure- } \\
\text { ment. }\end{array}$ & $\begin{array}{l}\text { Type of lymph: mild } \\
31 \% \text {, moderate } \\
52 \% \text {, severe } 17 \% \\
\text { Early lymp } 35 \% \\
\text { Chronic lymph } \\
65 \% \\
\text { Group 2: } \\
\text { Age: } 63 \text { (13) yrs } \\
\text { No lymph nodes re- } \\
\text { moved: } 10 \text { (5) } \\
\text { Type of lymp: mild } \\
24 \%, \quad \text { moderate } \\
52 \% \text {, severe } 24 \% \\
\text { Early lymph: } 48 \% \\
\text { Chronic lymph: } \\
52 \%\end{array}$ & & & \\
\hline $\begin{array}{l}\text { Sitzia } 2002 \\
\text { UK }\end{array}$ & $\begin{array}{l}2 \text { week parallel } \\
\text { group trial. } \\
\text { Randomisation } \\
\text { by computer-gener- } \\
\text { ated code managed } \\
\text { by non-clinical re- } \\
\text { searcher. } \\
\text { Two groups } \\
\text { Group 1: MLD } \\
\text { (n=15); } \\
\text { Group } 2 \text { : SLD } \\
\text { (n=13). } \\
\text { Withdrawn/ } \\
\text { excluded: } \\
1 \text { pt from Group } 2 \\
\text { only treated for } 5 \\
\text { days. } \\
\text { No adverse events }\end{array}$ & $\begin{array}{l}\text { Re- } \\
\text { cruited from all new } \\
\text { referrals to l'oedema } \\
\text { clinic from Jan '96 } \\
\text { to June '99. } \\
\text { All were } \\
\text { female, with unilat- } \\
\text { eral upper } \\
\text { limb oedema fol- } \\
\text { lowing trt for breast } \\
\text { ca. } \\
95 \text { referred; } \\
40 \text { fulfilled inclu- } \\
\text { sion criteria; } \\
12 \text { declined; } \\
28 \text { consented. } \\
\text { Group } 1 \\
\text { Mean (SD): } \\
\text { age = 68 (10.8) } \\
\% \text { Excess Volume at } \\
\text { start = 68.3 (39.5) } \\
\text { No. (\%) } \\
\text { Mastectomy \& ax- }\end{array}$ & $\begin{array}{l}\text { Group 1: } \\
\text { MLD given for } 40 \text { - } \\
80 \text { mins } 5 \text { days a } \\
\text { week for } 2 \text { weeks; } \\
\text { Group 2: } \\
\text { SLD performed by } \\
\text { same therapist } 20 \\
\text { mins } 5 \text { days a week } \\
\text { for } 2 \text { weeks } \\
\text { Following mas- } \\
\text { sage limbs in both } \\
\text { groups were ban- } \\
\text { daged using multi- } \\
\text { layer technique }\end{array}$ & $\begin{array}{l}\text { - \% Reduction in ex- } \\
\text { cess limb volume at } \\
2 \quad \text { weeks. } \\
\text { - Limb volume cal- } \\
\text { culated from surface } \\
\text { measurements. }\end{array}$ & $\begin{array}{l}\text { Trial does not follow } \\
\text { patients up for long } \\
\text { enough (i.e. for at } \\
\text { least } 6 \text { months) }\end{array}$ \\
\hline
\end{tabular}


Table 1. Details of excluded trials (Continued)

\begin{tabular}{|c|c|c|c|c|c|}
\hline & & $\begin{array}{l}\text { illary clearance = } \\
11(73) \\
\text { Lumpectomy \& ax- } \\
\text { illary clearance = } 2 \\
(13) \\
\text { Fine needle aspira- } \\
\text { tion = } 1(7) \\
\text { No surgery = } 1(7) \\
\text { Radiotherapy = } 13 \\
\text { (87) } \\
\text { Group } 2 \\
\text { Mean (SD): } \\
\text { age = 75 (10.2) } \\
\% \text { Excess Volume at } \\
\text { start = 58.5 (26.7) } \\
\text { No. (\%) } \\
\text { Mastectomy \& ax- } \\
\text { illary clearance = } \\
11(85) \\
\text { Lumpectomy \& ax- } \\
\text { illary clearance = } 2 \\
(15) \\
\text { Fine needle aspira- } \\
\text { tion = } 0 \\
\text { No surgery = } 0 \\
\text { Radiotherapy = } 10 \\
(77)\end{array}$ & & & \\
\hline $\begin{array}{l}\text { Szuba } 2002 \\
\text { USA }\end{array}$ & $\begin{array}{l}6 \text { month crossover } \\
\text { trial. } \\
\text { Randomisation } \\
\text { method not stated. } \\
\text { First phase of study: } \\
10 \text { day RCT with } 30 \\
\text { day follow up of IPC } \\
+ \text { DLT vs DLT. } \\
\text { Sec- } \\
\text { ond phase of study: } \\
2 \text { month crossover } \\
\text { RCT with } 6 \text { month } \\
\text { follow up of IPC + } \\
\text { DLT vs DLT. } \\
2 \text { groups: } \\
\text { (1) } \\
\text { mittent pneumatic }\end{array}$ & $\begin{array}{l}\text { Re- } \\
\text { cruitment from pa- } \\
\text { tients presenting to } \\
\text { Stanford Centre for } \\
\text { Lymphatic and Ve- } \\
\text { nous Disorders with } \\
\text { stable treated lym- } \\
\text { phoedema. } \\
\text { Lymphoedema was } \\
\text { defined as presence } \\
\text { of an increase of at } \\
\text { least } 20 \% \text { in the vol- } \\
\text { ume of the swollen } \\
\text { limb compared to } \\
\text { the volume of the } \\
\text { contralateral arm. } \\
\text { Demographic } \\
\text { data not reported by }\end{array}$ & $\begin{array}{l}\text { All participants had } \\
\text { completed an initial } \\
\text { course of intensive } \\
\text { DLT btwn } 1 \text { and } 12 \\
\text { months prior to en- } \\
\text { rolment. } \\
\text { (1) IPC, } 1 \text { hr daily } \\
\text { administered } \\
\text { at home + daily DLT } \\
\text { (MLD for } 30 \text { to } \\
60 \text { minutes (Vodder } \\
\text { type), compressive } \\
\text { bandaging and de- } \\
\text { congestive exercises } \\
\text { (2) Daily DLT alone }\end{array}$ & $\begin{array}{l}\text { - Arm volume (as- } \\
\text { sessed by water dis- } \\
\text { placement method) } \\
\text { - Tissue tonometry } \\
\text { (to assess elasticity } \\
\text { of the skin) } \\
\text { - Goniometry (to } \\
\text { measure joint mo- } \\
\text { bility) }\end{array}$ & $\begin{array}{l}\text { All participants were } \\
\text { free to con- } \\
\text { tinue with IPC after } \\
2 \text { months of treat- } \\
\text { ment, so assessment } \\
\text { at } 6 \text { month follow } \\
\text { up was not of ran- } \\
\text { domised groups. }\end{array}$ \\
\hline
\end{tabular}


Table 1. Details of excluded trials (Continued)

\begin{tabular}{|c|c|c|c|c|c|}
\hline & $\begin{array}{l}\text { compression (IPC) } \\
\text { + decongestive lym- } \\
\text { phatic } \\
\text { therapy (DLT) first, } \\
\text { DLT alone second, } \\
\mathrm{n}=13 \\
\text { (2) DLT alone first, } \\
\text { IPC + DLT second, } \\
\mathrm{n}=12 \\
\text { (Each participant } \\
\text { had both treatments } \\
\text { in a random order) } \\
\text { Excluded/ } \\
\text { withdrew: } \\
2 / 27 \text { did not com- } \\
\text { plete the study. After } \\
\text { the } 2 \text { month RCT, } \\
20 / 25 \text { continued us- } \\
\text { ing IPC and } 19 \text { were } \\
\text { still using IPC at } \\
\text { end of follow up. }\end{array}$ & $\begin{array}{l}\text { group. } \\
\text { All participants: } \\
\text { Age: } 65.9 \text { ( } 43 \text { to } 81) \\
\text { yrs } \\
\text { Average duration of } \\
\text { lymph: } 60 \text { (3 to } \\
\text { 480) mths } \\
\text { Average time from } \\
\text { surgery: } 113.7 \mathrm{mths}\end{array}$ & & & \\
\hline $\begin{array}{l}\text { Wilburn } 2006 \\
\text { USA }\end{array}$ & $\begin{array}{l}6 \text { week crossover } \\
\text { trial. } \\
\text { Randomisation } \\
\text { method not stated. } \\
2 \text { groups: } \\
\text { (1) Flexitouch (me- } \\
\text { chanical de- } \\
\text { vice which simulates } \\
\text { MLD) first, massage } \\
\text { second, n=5 } \\
\text { (2) Massage first, } \\
\text { Flexitouch second, } \\
\mathrm{n}=5 \\
\text { All participants used } \\
\text { a compression gar- } \\
\text { ment daily } \\
\text { Withdrawn/ } \\
\text { excluded: } \\
\text { There do not ap- } \\
\text { pear to be any with- } \\
\text { drawals. }\end{array}$ & $\begin{array}{l}\text { Re- } \\
\text { cruitment from pa- } \\
\text { tients presenting to } \\
\text { Stanford Centre for } \\
\text { Lymphatic and Ve- } \\
\text { nous Disorders. } \\
\text { Lymphoedema de- } \\
\text { fined as an increase } \\
\text { of at least } 10 \% \text { in } \\
\text { the mea- } \\
\text { sured volume of the } \\
\text { affected arm when } \\
\text { compared with the } \\
\text { contralateral limb. } \\
\text { Demographic } \\
\text { data not reported by } \\
\text { group. } \\
\text { All participants: } \\
\text { Age: } 60 \text { (7) yrs } \\
\text { Duration since ini- } \\
\text { tial cancer Rx: } 103 \\
\text { (87) mths } \\
\text { On- } \\
\text { set of arm swelling }\end{array}$ & $\begin{array}{l}\text { (1) Flexitouch self } \\
\text { administered for } 1 \\
\text { hour daily for } 14 \\
\text { consecutive days } \\
\text { (2) Self adminis- } \\
\text { tered massage for } 1 \\
\text { hour daily for } 14 \\
\text { consecutive days } \\
1 \text { week washout pe- } \\
\text { riod between treat- } \\
\text { ments where the use } \\
\text { of the compression } \\
\text { garment only was } \\
\text { permitted. }\end{array}$ & $\begin{array}{l}\text { - Limb volume (as- } \\
\text { sessed by surface } \\
\text { measurements and } \\
\text { a simplified formula } \\
\text { for a ftrustum) } \\
\text { - SF36 }\end{array}$ & $\begin{array}{l}\text { Trial does not follow } \\
\text { patients up for long } \\
\text { enough (i.e. for at } \\
\text { least } 6 \text { months). }\end{array}$ \\
\hline
\end{tabular}


Table 1. Details of excluded trials (Continued)

\begin{tabular}{|c|c|c|c|c|c|}
\hline & & $\begin{array}{l}\text { prior to enrolment: } \\
34 \text { (34) mths } \\
\text { Weight: } 75 \text { (12) kg }\end{array}$ & & & \\
\hline $\begin{array}{l}\text { Williams } 2002 \\
\text { UK }\end{array}$ & $\begin{array}{l}9 \text { week crossover } \\
\text { trial. } \\
\text { Method of } \\
\text { randomisation not } \\
\text { stated } \\
\text { Two trial groups } \\
\text { Group 1: MLD first } \\
\text { self-administered } \\
\text { massage [SAM] sec- } \\
\text { ond (no=15) } \\
\text { Group 2: SAM first, } \\
\text { MLD second } \\
\text { (n=16) } \\
\text { Withdrawn/ } \\
\text { excluded: } \\
\text { 2/31 both from } \\
\text { Group 2, } 1 \text { had pul- } \\
\text { monary embolus, } 1 \\
\text { herpes zoster in af- } \\
\text { fected area. }\end{array}$ & 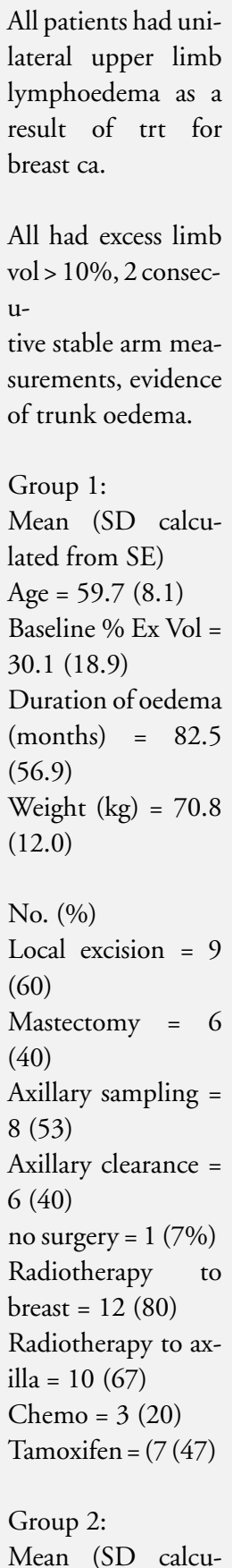 & $\begin{array}{l}\text { Group 1: } \\
15 \mathrm{x} 1 \text { hour ses- } \\
\text { sions of MLD over } \\
3 \text { weeks, performed } \\
\text { by therapists fol- } \\
\text { lowed by } 6 \text { weeks } \\
\text { washout period of } \\
\text { no trt, then } 3 \text { weeks } \\
\text { of } 20 \text { mins daily } \\
\text { SAM. } \\
\text { Group } 2 \text { : } \\
3 \text { weeks of } 20 \text { mins } \\
\text { daily } \\
\text { SAM. followed by } 6 \\
\text { weeks washout pe- } \\
\text { riod of no trt, then } \\
15 \times 1 \text { hour ses- } \\
\text { sions of MLD over } \\
3 \text { weeks, performed } \\
\text { by therapists. } \\
\text { Both } \\
\text { groups wore com- } \\
\text { pression sleeves dur- } \\
\text { ing the day through- } \\
\text { out the study. }\end{array}$ & $\begin{array}{l}\text { Changes in excess } \\
\text { limb volume. Vol- } \\
\text { ume measured elec- } \\
\text { tronically by Perom- } \\
\text { eter and also cal- } \\
\text { culated from sur- } \\
\text { face measurements } \\
\text { taken by hand. } \\
\text { Changes in caliper } \\
\text { creep on right and } \\
\text { left axillary folds, } \\
\text { measured by modi- } \\
\text { fied Harpenden skin } \\
\text { calipers. } \\
\text { Changes in dermal } \\
\text { depth on affected } \\
\text { side, measured by } \\
\text { skin ultrasound. } \\
\text { Changes in quality } \\
\text { of life measured by } \\
\text { EORTC QLQ C30 } \\
\text { Changes in move- } \\
\text { ment \& function } \\
\text { jective assessment of } \\
\text { change in areas of fi- } \\
\text { brosis. } \\
\text { sessment tool. } \\
\text { Subjective as- } \\
\text { sessment of changes } \\
\text { in altered sensation. } \\
\text { Change in arm and } \\
\text { trunk swelling } \\
\text { assessed subjectively } \\
\text { by "pinch test". } \\
\text { atided as- }\end{array}$ & $\begin{array}{l}\text { Trial does not follow } \\
\text { patients up for long } \\
\text { enough (i.e. for at } \\
\text { least } 6 \text { months) }\end{array}$ \\
\hline
\end{tabular}


Table 1. Details of excluded trials (Continued)

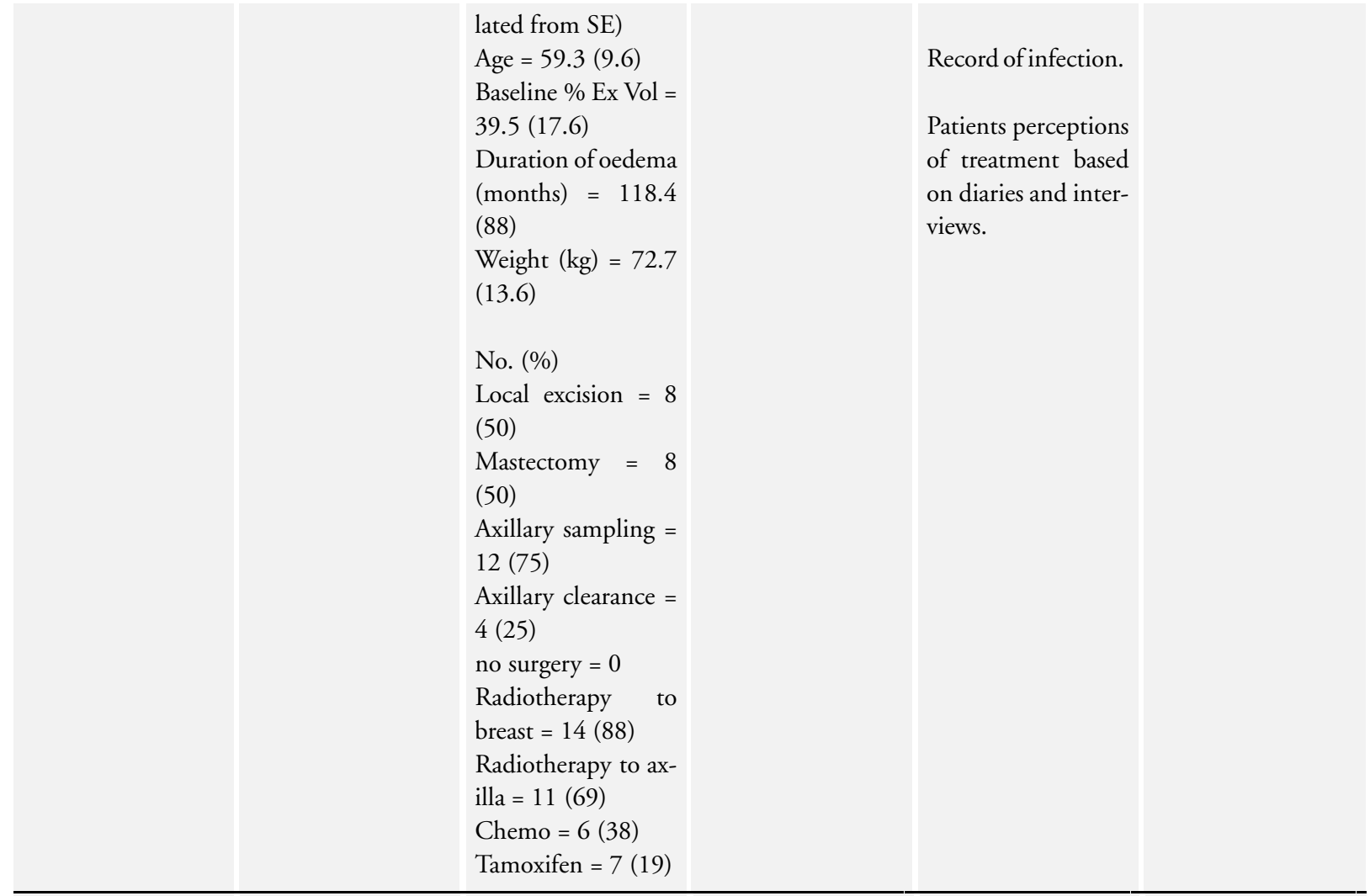

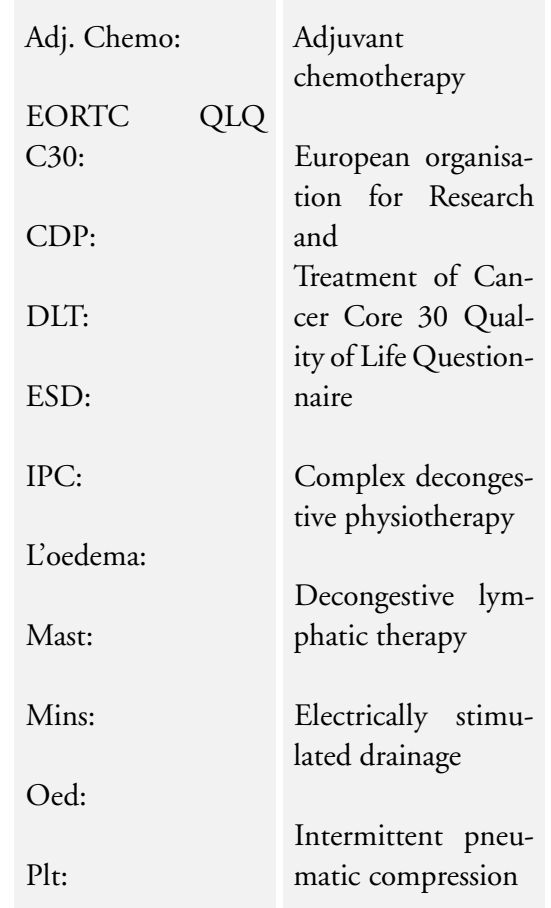


Table 1. Details of excluded trials (Continued)

\begin{tabular}{|l|l|}
\hline q: & Lymphoedema \\
\hline SAM: & Mastectomy \\
\hline SD: & Minutes \\
\hline SE: & Oedema \\
\hline SLD: & P \\
\hline SP & Self administered \\
\hline Surg: & massage \\
\hline Trt: & Standard deviation \\
\hline V: & Standard error \\
\hline & $\begin{array}{l}\text { Simple } \\
\text { drainage }\end{array}$ \\
\hline & $\begin{array}{l}\text { Standard } \\
\text { physiotherapy }\end{array}$ \\
\hline & Surgery \\
\hline & Treatment \\
\hline & \\
\hline &
\end{tabular}

Details of the included studies

The three eligible studies included a total of 150 participants. A summary of the participants, interventions and outcomes can be found in the Table of Included Studies and are also detailed here.

\section{Manual Lymph drainage study (MLD)}

Andersen 2000 designed a 12 month parallel group trial, with crossover from the control group to the MLD group after three months, if participants felt their response was unsatisfactory.

All the participants were women with unilateral lymphoedema of the upper limb following treatment for breast cancer. Patients with recurrent cancer or who were receiving treatment for lymphoedema in the three months preceding the trial were excluded. Women with an excess limb volume of more than $30 \%$ were excluded and offered intensive treatment with bandages but, rather confusingly, those who did not want the intensive treatment were allowed back into the trial. The investigators defined the minimum level of oedema for inclusion not as the percentage excess volume but as an absolute excess volume of more than $200 \mathrm{mls}$.

Participants were randomised to MLD followed by self-administered massage or no massage at all. The MLD group received eight sessions of MLD in two weeks and training in self-massage to be done daily for the remainder of the 12 month trial. Both control and MLD groups wore flat-knit compression sleeves daily, were 
instructed in exercises aimed at enhancing lymph flow and in skin care and prevention of infection.

The main outcome was a change in the percentage excess limb volume. Volume was calculated from surface measurements using "piecewise quadratic approximation, known as Simpson's rule of integration". The authors decided on a very complex approach to analysis of the data on volume. The investigators also assessed shoulder mobility on two planes: extension-flexion and adduction-abduction and asked patients to complete a questionnaire on symptoms related to lymphoedema and the European Organisation for Research and Treatment of Cancer (EORTC QLQ C30) questionnaire.

\section{Hosiery Study}

Hornsby 1995 investigated the use of hosiery on its own, rather than in conjunction with another therapy such as MLD.

The study was described as a randomised trial but the method of randomisation was not stated nor was the length of the trial. It was of a parallel group design with two study groups.

Participants were recruited from patients attending a local followup breast clinic, who, if they complained of swelling in the arm, were referred on to a lymphoedema clinic set up especially for the trial.

Both groups were taught exercises, skin care and self-administered massage. The experimental group were fitted with elastic compression sleeves that were worn day and night.

The description of the method for assessing the main outcome, limb volume, is a little confusing. It would appear that limb volume was measured by water displacement in two stages, level with a mark $10 \mathrm{cms}$ below the olecranon and then level with a mark $15 \mathrm{cms}$ above the olecranon. The normal limb volume at $15 \mathrm{cms}$ above the olecranon was subtracted from that of the swollen limb and recorded at each visit. It is not clear why two marks were needed or whether the final measurement was a combined total or was minus the volume up to the level of the first mark. At each visit participants were asked how much their swollen limb interfered with daily activities and whether they had any pain.

\section{Bandage Study}

Badger 2000 investigated multi-layer bandaging. The trial took place in London and involved two of the authors of this review.

The trial was described as randomised and the method of randomisation was reported. There were two trial groups running in parallel and the trial lasted six months.

Participants were recruited from the patients attending the lymphoedema clinic and included those with unilateral lymphoedema of upper or lower limb, primary in origin as well as lymphoedema secondary to the treatment of cancer.
Participants in the bandage group received a 19 day course of multi-layer bandaging and were then fitted with hosiery which was worn daily for the remainder of the trial. Participants in the hosiery alone group wore hosiery daily from the start of the trial. Both groups received advice on skin care, were taught exercises and self-administered massage (SAM) which they were asked to perform daily.

The main outcome was reduction in excess volume that was either calculated from surface measurements or measured electronically using a Perometer(r). Body weight was also noted. Instances of infection and venous thrombosis were noted throughout the trial.

\section{Risk of bias in included studies}

Methodological details of the three included studies (Andersen 2000; Badger 2000; Hornsby 1995) can be found in the table 'Characteristics of Included Studies'. These studies were also assessed for risk of bias (Higgins 2007) (see Risk of Bias tables for each study).

\section{Randomisation method}

The method of randomisation was not described in 2 studies ( Andersen 2000; Hornsby 1995). In the other study, random allocation was by a centralised telephone system (Badger 2000).

\section{Allocation concealment}

None of the included studies provided details of allocation concealment.

\section{Blinding}

None of the included studies appeared to be blinded.

\section{Incomplete data}

All of the studies had withdrawals and loss to follow up after randomisation, but in one study (Badger 2000), this proportion was not considered great enough to cause major bias in the results. In this study, of the 90 patients randomised, seven were excluded or withdrawn, four from the bandage plus hosiery group and three from the hosiery alone group. One participant declined treatment with bandages, two did not complete the course of bandaging, one developed a recurrence of cancer, one insisted on regular MLD and two never attended for follow-up. In the Anderson study, 2/44, one from each trial group, were withdrawn because they were found to be ineligible. After three months, 10 participants in the control group crossed over to the MLD group. A further five participants were excluded: one died of a heart attack, two developed a recurrence of cancer, one withdrew due to her husband's illness and one participant withdrew due to depression. In the Hornsby study, no explicit report was given of the withdrawals or exclusions from the 25 participants. It is clear from the data table included in the paper that the number of patients in the control group had dropped by about half by the third visit $(6 / 11$ remained) and by the fourth only one participant remained. No reasons were provided for this reduction in numbers. In the treatment group, follow up went on for eight visits but there was a steady falling off of participants from the third visit. Out of 14 participants at the start of the trial, data are available for only three by the end; again no reasons are 
put forward for this reduction in numbers.

\section{Selective outcome reporting}

None of the included studies provided details of their study protocols, so selective outcome reporting cannot be excluded.

\section{Other bias}

One study (Hornsby 1995) did not provide details of the comparability of randomised groups at baseline. For the other 2 studies, there was no evidence of a difference in prognostic factors at baseline.

\section{Effects of interventions}

Manual Lymph Drainage Study (Andersen 2000)

Of the 42 participants included in the analysis 22 were randomised to MLD + hosiery and 20 to hosiery alone. Other than endocrine therapy, received by 10 participants in the hosiery alone group and only two in the MLD group, the groups were evenly balanced in other respects such as age, duration of oedema, at the start of the trial.

The median excess volume at the start of the trial is reported as the absolute volume rather than as a percentage so it is difficult to judge how bad the participants' oedema was. By the end of the trial, the mean percentage reduction in excess limb volume was $60 \%(95 \%$ CI 43 to $78 \%)$ in the hosiery alone group as against $48 \%$ (95\% CI 32 to $65 \%$ ) in the MLD group. This difference was not significant. No differences were found between the two groups in the symptom scores.

The authors concluded that improvements seen in both groups were attributable to the use of compression sleeves and that MLD provided no extra benefit at any point during the trial.

Hosiery Study (Hornsby 1995)

There are problems with the reporting of this trial. From the text, it seems that the data in the tables represent the difference between swollen and normal limb volume at each visit. The raw data for each participant are tabulated but no means, standard deviations or confidence intervals are calculated. It is possible to work out the percentage reduction in excess limb volumes for each participant from these data. But the drop out rate is so high, particularly in the control group, that little information is available beyond the second month in the treated group and beyond the first month in the control group. Data concerning pain and how much swelling interfered with daily activities are not reported in any detail and not according to trial group.

The author concluded that, although this was a small study, the results suggest that wearing a compression sleeve is beneficial and that the high dropout rate in the control groups may have reflected the subjects' lack of progress.

Bandage Study (Badger 2000)

Data were available for analysis on 83 participants, 34 in the bandage + hosiery group and 49 in the hosiery alone group. Around two thirds of participants had upper limb oedema and most were female. There was a satisfactory balance between the groups in terms of age, duration of oedema, site, gender, body weight and excess volume at the start.

Both groups achieved a reduction in percentage of excess limb volume but that achieved by the bandage + hosiery group was consistently greater than that of the hosiery alone group. On day 19 , marking the end of the course of bandaging, the bandage + hosiery group achieved a mean reduction of $33.5 \%$ (SD 16.9) as against $9.6 \%$ (SD 20.4) in the hosiery alone group. Progress at week 12 was maintained at week 24 in both groups but that achieved by the bandage + hosiery group (mean $32.6 \%$ SD 33.2) was almost double that of the hosiery alone group (19.6\% SD 28.5).

The authors concluded that in this mixed group of patients bandage + hosiery resulted in a greater reduction in excess limb volume than hosiery alone and this difference in reduction was maintained long-term.

\section{DISCUSSION}

Any oedema of the surface tissues that involves a fluid component is likely to be influenced by the application of external pressure, as clinical experience world-wide has demonstrated over many years. From a physiological point of view, difficulties are likely to arise if fluid is trapped in fatty tissues (since fat absorbs pressure) or in fibrotic tissues where it becomes difficult for fluid to be displaced. Both of these scenarios are common in lymphoedema but despite these problems few therapists specialising in the management of lymphoedema doubt that improvements can be obtained through treatment. The question is not so much 'can lymphoedema be treated?' but rather 'what treatments reduce swelling, and the morbidity associated with swelling, most effectively?' In addition to this question, we need to ask 'what treatments produce lasting improvements?' since short-term improvements satisfy no one.

It appears that at present there is no drug or surgery that will reduce chronic oedema and allow the reduction to be maintained. Physical therapies remain the most commonly used treatments for lymphoedema and are usually combined in a treatment programme, since the general view is that no one treatment is likely to be successful on its own. The difficulty lies in establishing which of these physical treatments plays the most critical part in reducing and controlling swelling and which, if any, can be safely left out of the treatment programme.

This systematic review extends the findings of earlier nonCochrane systematic reviews of physical therapies for lymphoedema (Harris 2001; Kligman 2004; Moseley 2007). All reviews were limited by the lack of adequately designed randomised controlled trials. Most of the studies conducted so far in this field are either designed poorly or are poorly reported. Most are too small and provide too little follow-up to be of any use. There is a tendency to concentrate on one section of the lymphoedema 
population (i.e. breast cancer patients) when the growing body of evidence on prevalence and incidence suggests that lower limb oedema, either of primary origin or secondary to cancer and other conditions, is also a significant problem.

Our objective in this review was to assess the impact of physical treatment programmes on the volume, shape, condition and longterm control of oedema in lymphoedematous limbs and in addition to establish the psycho-social benefits of physical treatment. We were not able to answer any questions relating to the effect of treatment on the incidence of infection. While it is accepted that assessing the effect of treatment on the patients' psycho-social well-being presents considerable challenges there is a significant body of literature on this subject, particularly in relation to other chronic conditions such as diabetes and rheumatoid arthritis, that could be drawn upon. The few studies included in this review offer little to increase our understanding in this area.

\section{A U THORS, CONCLUSIONS}

\section{Implications for practice}

Of the three trials included here, only one studied lower as well as upper limb oedema. All three trials have their limitations and have yet to be replicated, so their results must be viewed with caution. There is weak evidence to support the use of multi-layer bandaging over hosiery alone.

\section{Implications for research}

There is a clear and pressing need for well-designed, randomised trials of the whole range of physical therapies if the best approach to managing lymphoedema is to be determined. Trials of complex physical therapy programmes, while not easy to conduct, are nevertheless possible.

\section{ACKNOW LEDGEMENTS}

Our thanks go to the members of the editorial office of the Cochrane Breast Cancer Group for assisting in all stages of the review and especially to Sharon Parker (Review Group Coordinator) and Nicole Holcroft and Joanne Talenta (Trials Search Coordinator).

Thanks are also expressed to the study investigators who provided additional information and were generally helpful.

We acknowledge the contribution of Anne Lethaby and Julie Brown (Cochrane updating project) for the 2008 update.

\section{REFERENCES}

\section{References to studies included in this review}

Andersen 2000 \{published data only\}

* Andersen L, Hojris I, Erlandsen M, Andersen J. Treatment of breast-cancer-related lymphedema with or without manual lymphatic drainage--a randomized study. Acta Oncologica 2000;39 (3):399-405.

Badger 2000 \{published data only\}

* Badger CMA, Peacock JL, Mortimer PS. A randomized, controlled, parallel-group clinical trial comparing multilayer bandaging followed by hosiery versus hosiery alone in the treatment of patients with lymphedema of the limb. Cancer 2000;88(12): 2832-7.

Hornsby 1995 \{published data only\}

* Hornsby R. The use of compression to treat lymphoedema. Professional Nurse 1995;11(2):127-8.

\section{References to studies excluded from this review}

Ahmed 2006 \{published data only\}

Ahmed RL, Thomas W, Yee D, Schmitz KH. Randomized controlled trial of weight training and lymphedema in breast cancer survivors. Journal of Clinical Oncology 2006;24(18):2765-2772.
Bertelli 1991 \{published data only\}

Bertelli G, Venturini M, Forno G, Macchiavello F, Dini D. Conservative treatment of postmastectomy lymphedema: a controlled randomised trial. Annals of Oncology 1991;2:575-8.

Didem 2005 \{published data only\} Didem K, Ufuk YS, Serdar S, Zumre A. The comparison of two different physiotherapy methods in treatment of lymphedema after breast surgery. Breast Cancer Research and Treatment 2005;93(1): $49-54$.

Dini 1998 \{published data only\}

Dini D, Del Mastro L, Gozza A, Lionetto R, Garrone O, Forno G, et al. The role of pneumatic compression in the treatment of postmastectomy lymphedema. A randomized phase III study. Annals of Oncology 1998;9:187-90.

Irdesel 2007 \{published data only\}

Irdesel J, Kahram an Celiktas S. Effectiveness of exercise and compression garments in the treatment of breast cancer related lymphedema. Turkiye Fiziksel Tip ve Rehabilitasyon Dergisi 2007;53 (1):16-21.

Johansson 1998 \{published data only\} Johansson K, Lie E, Ekdahl C, Lindfeldt J. A randomized study comparing manual lymph drainage with sequential pneumatic 
compression for treatment of postoperative arm lymphedema. Lymphology 1998;31(2):56-64.

Johansson 2005 \{published data only\}

Johansson K, Tibe K, Weibull A, Newton RC. Low intensity resistance exercise for breast cancer patients with arm lymphedema with or without compression sleeve. Lymphology 2005;38(4): 167-180.

McKenzie 2003 \{published data only\} McKenzie DC, Kalda AL. Effect of upper extremity exercise on secondary lymphedema in breast cancer patients: a pilot study. Journal of Clinical Oncology 2003;21(3):463-466.

McNeely 2004 \{published data only\}

McNeely ML, Magee DJ, Lees AW, Bagnall KM, Haykowsky M, Hanson J. The addition of manual lymph drainage to compression therapy for breast cancer related lymphedema: a randomized controlled trial. Breast Cancer Research and Treatment 2004;86(2): 95-106.

Sitzia 2002 \{published data only\}

Sitzia J, Sobrido L, Harlow W. Manual lymphatic drainage compared with simple lymphatic drainage in the treatment of postmastectomy lymphoedema: a pilot randomised trial. Physiotherapy 2002;88(2):99-107.

Szuba 2002 \{published data only\}

Szuba A. Literature watch. The addition of manual lymph drainage to compression therapy for breast cancer related lymphedema: a randomized controlled trial. Lymphatic Research and Biology 2005;3 (1):36-41.

* Szuba A, Achalu R, Rockson SG. Decongestive lymphatic therapy for patients with breast carcinoma-associated lymphedema. Cancer 2002;95:2260-2267.

Wilburn 2006 \{published data only\} Wilburn O, Wilburn P, Rockson SG. A pilot, prospective evaluation of a novel alternative for maintenance therapy of breast cancer associated lymphedema. BMC Cancer 2006;6:84.

Williams 2002 \{published data only\}

Williams AF, Vadgama A, Franks PJ, Mortimer PS. A randomized controlled crossover study of MLD therapy in women with breast cancer-related lymphoedema.. European Journal of Cancer Care 2002;11(4):254-61.

\section{References to studies awaiting assessment}

\section{Bergan 1998 \{published data only\}}

Bergan JJ, Sparks S, Angle N. A comparison of compression pumps in the treatment of lymphedema.. Vascular Surgery 1998;32(5): 455-62.

Thiadens 1999 \{published data only\}

Thiadens S, Wright E. The effectiveness of compression sleeves in the maintenance of postmastectomy lymphedema. JOBST? Beiersdorf Website Pre 1999.

\section{References to ongoing studies}

\section{Didem 2008 \{unpublished data only\}}

Comparison of two different physiotherapy methods in treatment of lymphedema after breast surgery. Ongoing study Participants treated between June 2002 and May 2003.
Follow up at 6 months, 1, 2 and 3 years after treatment was initiated..

Schmitz 2006 \{unpublished data only\}

PAL (Physical Activity and Lymphedema) trial. Ongoing study Start date: October 2005.

Finish date: June 2008..

\section{Additional references}

\section{Badger 1988}

Badger CMA, Mortimer PS, Regnard CFB, Twycross RG. Pain in the chronically swollen limb. Progress in Lymphology - XI. Vienna: Elsevier Science Publishers, 1988:243-5.

\section{Badger 1997}

Badger C. Shape. Chapter in: A study of the efficacy of multilayer bandaging and compression hosiery versus hosiery alone in the management of lymphoedema of the limbs and their effects on the swollen limb. Unpublished Thesis, London 1997.

\section{Carroll 1992}

Carroll D, Rose KE. Treatment leads to significant improvement: effect of conservative treatment on pain in lymphoedema. Professional Nurse 1992;8(1):32-6.

\section{Erickson 2001}

Erickson VS, Pearson ML, Ganz PA, Adams J, Kahn KL. Arm edema in breast cancer patients. Journal of the National Cancer Institute 2001;93(2):96-111.

\section{Foldi 1985}

Foldi E, Foldi M, Weissleder H. Conservative treatment of lymphoedema of the limbs. Angiology 1985;36(3):171-80.

\section{Harris 2001}

Harris SR, Hugi MR, Olivotto IA, Levine M. Clinical practice guidelines for the care and treatment of breast cancer: 11 . Lymphedema. Canadian Medical Association Journal 2001;164(2): 191-199.

\section{Higgins 2007}

Higgins JPT, Altman DG (eds). Assessing risk of bias in included studies (Part of Chapter 8 - under consultation). Cochrane Handbook of Systematic Reviews of Interventions.

\section{Kligman 2004}

Kligman L, Wong RKS, Johnston M, Laetsch NS. The treatment of lymphedema related to breast cancer: a systematic review and evidence summary. Support Care Cancer 2004;12:421-431.

\section{Levick 1991}

Levick JR. An Introduction to Cardiovascular Physiology. First Edition. London: Butterworth, 1991.

\section{Moffatt 2003}

Moffatt CJ, Franks PJ, Doherty DC, Williams AF, BAdger C, Jeffs E, Bosanquet N, Mortimer PS. Lymphoedema: an underestimated health problem. Quarterly Journal of Medicine 2003;96(10):731-8.

\section{Mortimer 1995}

Mortimer PS. Managing Lymphoedema. Clinical and Experimental Dermatology 1995;20:98-106. 


\section{Moseley 2007}

Moseley AL, Carati CJ, Piller NB. A systematic review of common conservative therapies for arm lymphoedema secondary to breast cancer treatment. Annals of Oncology 2007;8(4):639-646.

\section{Petrek 1998}

Petrek JA, Heelan MC. Incidence of breast carcinoma-related lymphoedema. Cancer 1998;12 Supple:2776-81.

Sitzia 1997

Sitzia J, Stanton AWB, Badger C. A review of outcome indicators in the treatment of chronic limb oedema. Clinical Rehabilitation 1997;11:181-91

\section{Tobin 1993}

Tobin M, Lacey HJ, Meyer L, Mortimer PS. The psychological morbidity of breast cancer related arm swelling. Cancer 1993;72 (11):3248-52.

\section{References to other published versions of this review}

\section{Badger 2004}

Badger C, Preston N, Seers K, Mortimer P. Physical therapies for reducing and controlling lymphoedema of the limbs. Cochrane Database of Systematic Reviews 2004, Issue Issue 4. [Art. No.: CD003141. DOI: 10.1002/14651858.CD003141.pub2]

* Indicates the major publication for the study 
CHARACTERISTICS OF STUDIES

\section{Characteristics of included studies [ordered by study ID]}

Andersen 2000

\begin{tabular}{|c|c|}
\hline Methods & $\begin{array}{l}12 \text { month trial } \\
\text { Method of randomisation not reported } \\
2 \text { Trial Groups } \\
\text { Group 1: Standard treatment alone (no.23) } \\
\text { Group 2: Standard treatment + MLD (no.21) } \\
\text { Group } 1 \text { pts allowed to cross over to Group } 2 \text { after } 3 \text { months if not satisfied with response. } \\
\text { Withdrawn / excluded } \\
\text { no.7 out of total } 44 \text { : } \\
2 \text { / } 44 \text { pts ( } 1 \text { in each group) found to be ineligible: } \\
1 \text { had recurrent cancer; } \\
1 \text { less than } 4 \text { months post surgery; } \\
1 / 44 \text { withdrew due to husband's illness (? which group); } \\
1 / 44 \text { did not return at } 12 \text { months due to depression; } \\
1 / 44 \text { died } \\
\text { According to group: } \\
\text { Group 1: } \\
2 / 23 \text { local recurrence } \\
\text { Group 2: } \\
1 / 21 \text { died }\end{array}$ \\
\hline Participants & $\begin{array}{l}\text { Recruited breast ca. patients attending lymphoedema clinic } \\
44 \text { randomized } \\
\text { Group 1: n22 } \\
\text { [Median (range)] } \\
\text { Age 56yrs (29-77) } \\
\text { ExVol 361mls (78-1184) } \\
\text { Duration of oedema 12mnths (4-126) } \\
\text { Group 2: n20 } \\
\text { [Median (range)] } \\
\text { Age 53yrs (25-73) } \\
\text { ExVol 340mls (161-1297) } \\
\text { Duration of oedema 15mnths (5-183) } \\
\text { Breast Cancer treatment: } \\
\text { Group 1: } \\
\text { axillary dissection n11; } \\
\text { No RT n11; } \\
\text { RT including axilla n4; } \\
\text { RT not including axilla n7 } \\
\text { Group 2: } \\
\text { axillary dissection n15; } \\
\text { No RT n8; } \\
\text { RT including axilla n2; } \\
\text { RT not including axilla } 10\end{array}$ \\
\hline
\end{tabular}


Andersen 2000 (Continued)

\begin{tabular}{|c|c|c|}
\hline Interventions & \multicolumn{2}{|c|}{$\begin{array}{l}\text { Group } 1 \\
\text { Standard treatment consisted of compression sleeve worn during day - used decreasing } \\
\text { sizes for first } 2 \text { weeks then fitted with made-to-measure sleeves; } \\
\text { advice on exercises and skin care. } \\
\text { Group } 2 \\
\text { Standard treatment as above plus } 8 \text { sessions of MLD in } 2 \text { weeks; then taught SAM to be } \\
\text { used daily. }\end{array}$} \\
\hline Outcomes & \multicolumn{2}{|c|}{$\begin{array}{l}\text { Change in limb volume, calculated from surface measurements; } \\
\text { questionnaire on related symptoms and on compliance with treatment. } \\
\text { Assessments performed at start, } 3,6, \& 9 \text { months. } \\
\text { Further assessment at } 4 \text { months for any patients crossing over. }\end{array}$} \\
\hline Notes & \multicolumn{2}{|c|}{ Author contacted re method of randomisation - no response. } \\
\hline \multicolumn{3}{|l|}{ Risk of bias } \\
\hline Item & Authors' judgement & Description \\
\hline Adequate sequence generation? & Unclear & No description provided \\
\hline Allocation concealment? & Unclear & No description provided \\
\hline $\begin{array}{l}\text { Blinding? } \\
\text { All outcomes }\end{array}$ & No & Very unlikely for any outcomes \\
\hline $\begin{array}{l}\text { Incomplete outcome data addressed? } \\
\text { All outcomes }\end{array}$ & No & $\begin{array}{l}\text { Of a total of } 44 \text { participants, } 2 \text { were ini- } \\
\text { tially excluded because of ineligibility. Af- } \\
\text { ter } 3 \text { months, } 10 \text { participants crossed over } \\
\text { to the other treatment and a further } 5 \text { par- } \\
\text { ticipants were excluded. }\end{array}$ \\
\hline Free of selective reporting? & Unclear & Not clear \\
\hline Free of other bias? & Yes & No indication of any other likely bias \\
\hline
\end{tabular}




\begin{tabular}{|c|c|}
\hline Methods & $\begin{array}{l}6 \text { Month trial } \\
\text { Central telephone randomisation } \\
\text { Group 1: } \\
\text { Multi-Layer Bandaging [MLB] + Hosiery, (n38); } \\
\text { Group 2: } \\
\text { Hosiery alone, (n52). } \\
90 \text { patients randomised, } \\
\text { results reported on } 83 . \\
\text { Withdrawn/excluded: } \\
7 \text { in all } \\
\text { Group 1: } \\
1 \text { declined treatment; } \\
1 \text { developed recurrent Ca. } \\
1 \text { only had } 14 \text { days bandaging; } \\
1 \text { had only } 11 \text { days bandaging. } \\
\text { Group 2: } \\
1 \text { insisted on continuing MLD; } \\
2 \text { never attended for follow-up }\end{array}$ \\
\hline Participants & $\begin{array}{l}\text { Patients attending the Lymphoedema Service with unilateral lymphoedema; cancer- } \\
\text { related and non-cancer-related, affecting upper or lower limb with }>20 \% \text { excess volume. } \\
\text { Recruited over a } 2 \text { year period } \\
\text { Details of cancer treatment not reported } \\
\text { Group } 1 \\
\text { Mean (SD) } \\
\text { Age }=57.3 y r s ~(14.5) \\
\text { Duration of oedema = } 48 \text { months }(96) \\
\text { Baseline } \% \text { ExVol }=48.6 \%(25.6) \\
\text { Weight }=73 \mathrm{Kgs}(14) \\
\text { N }(\%) \\
\text { Male }=3(9) \\
\text { Female }=31(91) \\
\text { Upper limb }=21(62) \\
\text { Lower limb }=13(38) \\
\text { Group } 2 \\
\text { Mean }(S D) \\
\text { Age }=57.4 \text { yrs }(14.6) \\
\text { Duration of oedema }=60 \text { months }(96) \\
\text { Baseline } \% \text { ExVol }=41.9 \%(25.6) \\
\text { Weight }=71 \mathrm{Kgs}(13) \\
\text { N }(\%) \\
\text { Male }=4(8) \\
\text { Female }=45(92) \\
\text { Upper limb }=33(67) \\
\text { Lower limb }=16(33)\end{array}$ \\
\hline Interventions & $\begin{array}{l}\text { Group 1: } \\
18 \text { day course of daily MLB kept in place around the clock, followed by compression } \\
\text { hosiery worn during the day for remainder of trial } \\
\text { Group 2: }\end{array}$ \\
\hline
\end{tabular}


Compression hosiery alone, worn daily throughout the whole of trial

Both groups were asked to exercise daily and perform SAM daily and advised on daily skin care.

Outcomes

$\%$ Reduction / increase in excess limb volume calculated from surface measurements or from electronic measurements (Perometer)

Change in body weight

Notes

\section{Risk of bias}

\begin{tabular}{lll}
\hline Item & Authors' judgement & Description \\
\hline Adequate sequence generation? & Yes & $\begin{array}{l}\text { Method of randomisation was by cen- } \\
\text { tralised telephone system }\end{array}$ \\
\hline $\begin{array}{l}\text { Allocation concealment? } \\
\begin{array}{l}\text { Blinding? } \\
\text { All outcomes }\end{array}\end{array}$ & Unclear & No details reported \\
\hline $\begin{array}{l}\text { Incomplete outcome data addressed? } \\
\text { All outcomes }\end{array}$ & Yes & Blinding highly unlikely \\
\hline $\begin{array}{l}\text { Free of selective reporting? } \\
\text { Free of other bias? }\end{array}$ & Unclear & Dropouts not likely to cause major bias \\
\hline
\end{tabular}

Hornsby 1995

\begin{tabular}{ll} 
Methods & Length of trial not stated - follow-up appeared to continue for 7 months. \\
Method of randomisation not stated \\
Two trial groups: \\
Group1: Sleeve (n14) \\
Group 2: No sleeve (n11) \\
Withdrawn / excluded \\
By the end of the trial only 3 patients remained, all in Group 1. \\
No reason given for the dropouts other than to say it was presumably due to lack of \\
progress. \\
No adverse events reported \\
\hline Participants & $\begin{array}{l}\text { Patients with oedema attending F-Up breast clinic between Nov '91 to Dec' } 92 \text { referred } \\
\text { to a lymphoedema clinic set up for trial. } \\
\text { n60 referred; } \\
\text { n58 attended; } \\
25 \text { women consented to study } \\
\text { Not possible to say if groups are balanced as no details provided on: }\end{array}$ \\
\hline
\end{tabular}


Hornsby 1995 (Continued)

age;

duration of oedema;

breast cancer treatment;

severity of oedema at outset (not possible to calculate the \% Excess Volume at Start from data provided as normal limb volumes are not reported).

\begin{tabular}{|c|c|c|}
\hline Interventions & \multicolumn{2}{|c|}{$\begin{array}{l}\text { Both groups were taught exercises and self-administered massage and given advice on } \\
\text { skin care. } \\
\text { Group 1: } \\
\text { compression sleeves worn day and night; } \\
\text { Group 2: } \\
\text { no treatment. }\end{array}$} \\
\hline Outcomes & \multicolumn{2}{|c|}{$\begin{array}{l}\text { Reduction / increase in excess limb volume measured by water displacement every } 4 \\
\text { weeks } \\
\text { Patients were questioned about how much l'oedema interfered with daily living activities } \\
\text { at each visit. No information provided concerning the tool used. } \\
\text { Also questioned about pain at each visit. No information provided on the scale that was } \\
\text { used }\end{array}$} \\
\hline \multicolumn{3}{|l|}{ Notes } \\
\hline \multicolumn{3}{|l|}{ Risk of bias } \\
\hline Item & Authors' judgement & Description \\
\hline Adequate sequence generation? & Unclear & No details provided \\
\hline Allocation concealment? & Unclear & No details provided \\
\hline $\begin{array}{l}\text { Blinding? } \\
\text { All outcomes }\end{array}$ & No & Blinding highly unlikely \\
\hline $\begin{array}{l}\text { Incomplete outcome data addressed? } \\
\text { All outcomes }\end{array}$ & No & $\begin{array}{l}\text { Significant number of dropouts and no rea- } \\
\text { sons given. Out of } 14 \text { participants at the } \\
\text { start of the trial, data is available for only } 3 \\
\text { participants at the end. }\end{array}$ \\
\hline Free of selective reporting? & Unclear & No details provided \\
\hline Free of other bias? & Unclear & $\begin{array}{l}\text { No details provided of comparability of } \\
\text { groups at baseline }\end{array}$ \\
\hline
\end{tabular}

Ca: Cancer

Ex Vol: Excess Volume

MLD: Manual Lymph Drainage

N: Number

PCT: Pneumatic Compression Therapy 
RT: Radiotherapy

SAM: Self Administered Massage

SD: Standard Deviation

Characteristics of excluded studies [ordered by study ID]

\begin{tabular}{ll}
\hline Ahmed 2006 & $\begin{array}{l}\text { RCT comparing twice a week weight training with no weight training in } 45 \text { women with breast cancer associated } \\
\text { lymphoedema. Only a proportion of the participants (29\%) had lymphoedema at baseline - see Table } 1 \text { for details. }\end{array}$ \\
\hline Bertelli 1991 & $\begin{array}{l}\text { RCT comparing hosiery with electrically stimulated drainage versus hosiery alone in breast cancer associated } \\
\text { lymphoedema. Did not use limb volume as the method for assessing change in size - see Table } 1 \text { for details. }\end{array}$ \\
\hline Didem 2005 & $\begin{array}{l}\text { RCT with crossover design comparing a complex decongestive physiotherapy treatment (lymph drainage, multi } \\
\text { layer compression bandage, elevation, remedial exercises and skin care) with standard physiotherapy (bandage, } \\
\text { elevation, head-neck shoulder exercises and skin care) in } 53 \text { patients with breast cancer associated lymphoedema. } \\
\text { Follow up was at the end of the fourth week of treatment - did not satisfy eligibility criteria of at least } 6 \text { months. } \\
\text { The authors state that they are going to report long term follow up results in a further publication (see Ongoing } \\
\text { Studies). }\end{array}$
\end{tabular}

Dini 1998 RCT comparing pneumatic compression therapy (PCT) versus no PCT in 80 patients. Only lasted 9 weeks and did not use limb volume as the method for assessing change in size - see Table 1 for details

Irdesel 2007 RCT comparing exercise plus compression with exercise alone in 19 patients with breast cancer associated lymphoedema. The efficacy of treatment was assessed by measurement of the arm circumference rather than limb volume - see Table 1 for details.

Johansson 1998 RCT comparing manual lymph drainage versus sequential pneumatic compression in 28 patients. Only lasted 4 weeks - see Table 1 for details.

Johansson 2005 RCT comparing low intensity exercise plus compression sleeve with low intensity exercise alone in 31 patients with breast cancer related arm lymphoedema. Measurements were taken immediately after treatment and 24 hours later - see Table 1 for details.

McKenzie 2003 RCT comparing upper body exercise with no exercise in 14 patients with previous breast cancer. Treatment lasted for 8 weeks and follow up was every 2 weeks until the end of the study - see Table 1 for details.

McNeely 2004 RCT comparing manual lymph drainage plus compression with compression alone in 50 women with breast cancer related lymphoedema. Treatment and follow up lasted for 4 weeks - see Table 1 for details.

Sitzia 2002 RCT comparing manual lymph drainage and simple lymph drainage in 28 patients. Only lasted 2 weeks - see Table 1 for details.

Szuba 2002 RCT with crossover design comparing intermittent pneumatic compression plus decongestive lymphatic therapy with decongestive lymphatic therapy alone (MLD, compressive wrapping of the limb and decongestive exercises) in 27 patients with breast cancer associated lymphoedema. Follow up was for 6 months, but after 2 months of treatment, almost all the participants elected to take the experimental treatment - see Table 1 for details.

Wilburn 2006 RCT with crossover design comparing a mechanical device designed to simulate MLD (Flexitouch)with massage in 10 patients with breast cancer associated lymphoedema. Duration of trial was 2 weeks, 1 week washout and 2 more weeks with alternate treatment - see Table 1 for details. 
Williams 2002 RCT crossover trial comparing manual lymph drainage versus self-administered massage in 31 patients. Only lasted 9 weeks - see Table 1 for details.

MLD: Manual Lymph Drainage

RCT: Randomised Controlled Trial

Characteristics of ongoing studies [ordered by study ID]

\section{Didem 2008}

\begin{tabular}{|c|c|}
\hline Trial name or title & Comparison of two different physiotherapy methods in treatment of lymphedema after breast surgery \\
\hline \multicolumn{2}{|l|}{ Methods } \\
\hline Participants & $\begin{array}{l}\mathrm{n}=53 \text {. } \\
\text { Patients had developed unilateral lyphedema (mild-moderate) of duration at least } 1 \text { year after breast cancer } \\
\text { treatment. }\end{array}$ \\
\hline Interventions & $\begin{array}{l}\text { (1) Complex decongestive physiotherapy (manual lymph drainage, compression bandage, remedial exercises } \\
\text { and skin care. } \\
\text { (2) Standard physiotherapy (bandage, elevation, head-neck and shoulder exercises and skin care) }\end{array}$ \\
\hline Outcomes & $\begin{array}{l}\text { (1) Range of motion (extension-flexion, abduction-adduction, external rotation) } \\
\text { (2) Circumferential measurement of arm } \\
\text { (3) Volumetric measurement of arm (by water displacement) }\end{array}$ \\
\hline Starting date & $\begin{array}{l}\text { Participants treated between June } 2002 \text { and May } 2003 . \\
\text { Follow up at } 6 \text { months, 1, } 2 \text { and } 3 \text { years after treatment was initiated. }\end{array}$ \\
\hline Contact information & yuzbasioglu@deu.edu.tr \\
\hline Notes & $\begin{array}{l}\text { Longer follow up of patients given treatment for } 4 \text { weeks. Contact was made with the author who stated that } \\
\text { analysis was ongoing at longer follow up. }\end{array}$ \\
\hline
\end{tabular}

\section{Schmitz 2006}

Trial name or title PAL (Physical Activity and Lymphedema)trial

\begin{tabular}{ll}
\hline Methods & \\
\hline Participants & $\begin{array}{l}\mathrm{n}=288 \\
\text { Participants were } 1-15 \text { years after breast cancer diagnosis, currently free of cancer recruited in } 2 \text { strata: } \\
\text { (1) women with stable lymphedema (5-15 years post diagnosis) } \\
\text { (2) women without lymphedema (1-5 years post diagnosis). }\end{array}$
\end{tabular}


Schmitz 2006 (Continued)

\begin{tabular}{ll} 
Interventions & $\begin{array}{l}\text { (1) Exercise intervention of twice weekly strength training (13 weeks of supervised training (90 mins/session) } \\
\text { + 39 weeks of unsupervised training (90 mins/session)) } \\
\text { (2) Non exercising control group }\end{array}$ \\
\hline Outcomes & $\begin{array}{l}\text { (1) arm circumference } \\
\text { (2) extra-cellular water in the arm } \\
\text { (3) volumetry } \\
\text { (4) function tests (range of motion, pain, grip strength) }\end{array}$ \\
\hline Starting date & $\begin{array}{l}\text { Start date: October 2005. } \\
\text { Finish date: June 2008. }\end{array}$ \\
\hline Contact information & schmitz@mail.med.upenn.edu \\
\hline Notes & Contact made with author. Results forthcoming after completion of trial. \\
\hline
\end{tabular}




\section{DATA AND ANALYSES}

This review has no analyses.

\section{A P P E N D I CES}

Appendix I. Search strategy for CENTRAL (Issue I, 2008)

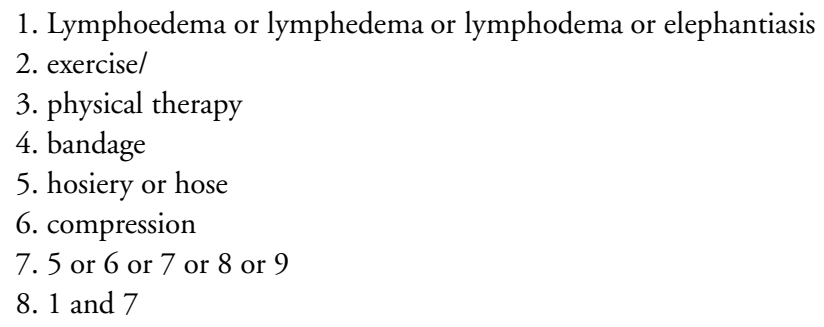

Appendix 2. Search strategy for CINAHL (Ovid) (1982 to February 2008)
1. Lymphedema/or elephantiasis/
2. Lymph?dema or elephantiasis
3. 1 or 2
4. Physical therapy/
5. Bandages and dressings/
6. Compression garments/
7. physical therapy
8. bandage
9. hosiery or hose
10. compression
11.4 or 5 or 6 or 7 or 8 or 9 or 10
12.3 and 11

Appendix 3. Search strategy for MEDLINE (Ovid) (1960 to February 2008)

1. Lymphedema/or elephantiasis/

2. Lymph?dema or elephantiasis

3. 1 or 2

4. Physical therapy modalities/

5. Bandages/

6. Intermittent pneumatic compression devices/

7. physical therapy

8. bandage

9. hosiery or hose

10. compression

11. 4 or 5 or 6 or 7 or 8 or 9 or 10

12. 3 and 11 
Appendix 4. Search strategy for EMBASE (Ovid) (I 980 to February 2008)

1. Lymphedema/or elephantiasis/

2. Lymph?dema or elephantiasis

3. 1 or 2

4. Physiotherapy/

5. Bandage/

6. Kinesiotherapy/

7. Intermittent pneumatic compression device/

8. Compression therapy/

9. physical therapy

10. bandage

11. hosiery or hose

12. compression

13. 4 or 5 or 6 or 7 or 8 or 9 or 10 or 11 or 12

14.3 and 13

Appendix 5. Search strategy for National Research Register (NRR) (February 2008)

1. lymphedema or lymphoedema or lymphodema

Appendix 6. Search strategy British Nursing Index, British Library Index, UnCover, PASCAL (September 2003)

Lymph?edema OR elephantiasis

a) AND physical therapy

b) AND bandage* OR (compression bandage*)

c) AND hosiery OR hose

d) OR hosiery OR hose NEAR compression

e) OR (compression stocking*) OR (compression sleeve*)

f) AND (pneumatic compression) OR (compression pump)

AND

g) (reduc* limb volume)

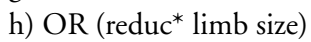

i) OR (reduc* excess volume)

j) OR (reduc* excess limb volume)

k) OR (reduc* oedema OR edema volume)

Lymph?edema OR elephantiasis

l) AND physical therapy

m) AND bandage*

n) AND (compression bandage*)

o) AND hosiery OR hose

p) AND (compression hosiery OR hose)

q) AND (compression stocking*) OR (compression sleeve*)

AND

r) (Quality of Life measure*) OR (Quality of Life tool*) 
WHAT'S NEW

Last assessed as up-to-date: 19 February 2008.

\begin{tabular}{|c|c|c|}
\hline 8 May 2008 & New search has been performed & $\begin{array}{l}\text { Updated } 2008 \text { as part of the Cochrane Updating Project. New search no change } \\
\text { to conclusions or citation. Risk of bias tables added }\end{array}$ \\
\hline 8 May 2008 & Amended & Converted to new review format. \\
\hline
\end{tabular}

\section{H I S T O R Y}

Protocol first published: Issue 3, 2001

Review first published: Issue 4, 2004

\begin{tabular}{l|l|l}
\hline 30 August 2004 & Amended & First review publication \\
\hline 30 May 2001 & Amended & Protocol publication \\
\hline
\end{tabular}

\section{CONTRIBUTIONSOFAUTHORS}

$\mathrm{CB}$ was the principal reviewer and wrote up the review. $\mathrm{CB}$ and NP were responsible for the extraction of the data. NP acted as the second reviewer and collated the final draft of the review. PM provided clinical direction and both KS and PM advised, and helped write both the protocol and the review.

In 2008, Anne Lethaby undertook to update the review as part of the Cochrane updating project. A new search was conducted and trials identified were independently scanned by Anne Lethaby and Julie Brown. No new trials were identified, but a number of potentially relevant trials were added to the Excluded Studies tables and two new ongoing trials were identified and documented. Anne Lethaby also assessed the included studies for risk of bias.

\section{DECLARATIONS OF INTEREST}

$\mathrm{CB}$ and $\mathrm{PM}$ are authors on one of the included trials. $\mathrm{CB}$ was the principal reviewer and wrote up the review.

\section{SOURCES OF SUPPORT}




\section{Internal sources}

- Royal College of Nursing, UK.

\section{External sources}

- No sources of support supplied

\section{NOTES}

The review was updated in 2008 as part of a pilot Cochrane updating project. No further trials met the inclusion criteria. A number of trials were added to the Excluded Studies Table and a Risk of Bias table was added.

\section{NDEX TERMS}

\section{Medical Subject Headings (MeSH)}

*Physical Therapy Modalities; Bandages; Lymphedema [*rehabilitation]; Randomized Controlled Trials as Topic

\section{MeSH check words}

Humans 